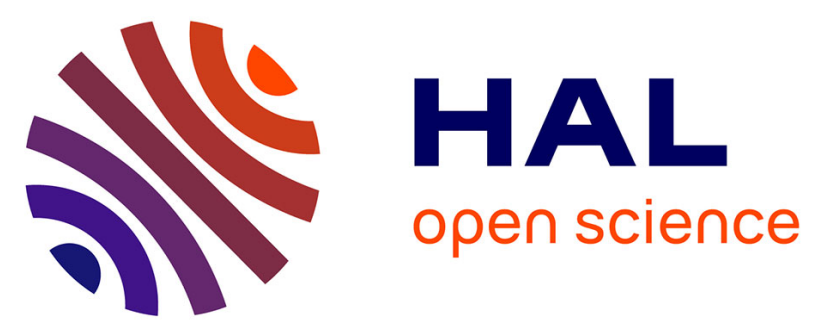

\title{
Genomic sequencing reveals historical, demographic and selective factors associated with the diversification of the fire-associated fungus Neurospora discreta
}

Pierre Gladieux, Benjamin Wilson, Fanny Perraudeau, Liliam Montoya, David Kowbel, Christopher Hann-Soden, Monika Fisher, Iman Sylvain, David Jacobson, John Taylor

\section{To cite this version:}

Pierre Gladieux, Benjamin Wilson, Fanny Perraudeau, Liliam Montoya, David Kowbel, et al.. Genomic sequencing reveals historical, demographic and selective factors associated with the diversification of the fire-associated fungus Neurospora discreta. Molecular Ecology, 2015, 24 (22), pp.5657-5675. 10.1111/mec.13417 . hal-01303142

\section{HAL Id: hal-01303142 \\ https://hal.science/hal-01303142}

Submitted on 11 May 2016

HAL is a multi-disciplinary open access archive for the deposit and dissemination of scientific research documents, whether they are published or not. The documents may come from teaching and research institutions in France or abroad, or from public or private research centers.
L'archive ouverte pluridisciplinaire $\mathbf{H A L}$, est destinée au dépôt et à la diffusion de documents scientifiques de niveau recherche, publiés ou non, émanant des établissements d'enseignement et de recherche français ou étrangers, des laboratoires publics ou privés. 


\section{Genomic Sequencing Reveals Historical, Demographic, and Selective factors Associated}

\section{With the Diversification of the Fire-Associated Fungus NeUROSPORA DISCRETA}

3 Pierre Gladieux ${ }^{1,2^{*}}$, Benjamin A Wilson ${ }^{3}$, Fanny Perraudeau ${ }^{1,4}$, Liliam A Montoya ${ }^{1}$, David Kowbel ${ }^{1}$,

4 Christopher Hann-Soden ${ }^{1}$, Monika Fischer ${ }^{1}$, Iman Sylvain ${ }^{1}$, David J Jacobson ${ }^{1}$, John W Taylor ${ }^{1}$

5

$6 \quad{ }^{1}$ Department of Plant and Microbial Biology, University of California, Berkeley, USA

$7 \quad{ }^{2}$ Ecologie Systematique Evolution, Université Paris Sud, Batiment 360, 91405 Orsay, France

$8{ }^{3}$ Department of Biology, Stanford University, USA

$9{ }^{4}$ Ecole Polytechnique, Route de Saclay, 91128 Palaiseau, France

10 *present address: INRA, UMR BGPI, TA A54/K, F-34398, Montpellier, France; CIRAD, F-34398, Montpellier, France

11

12 Correspondence: P Gladieux pierre.gladieux@supagro.inra.fr; UMR BGPI, TA A54/K, F-34398, Montpellier, France;

13 Phone +33 $499624863 ;$ Fax +33499624848

14

15

16 KeYWORDS: Ascomycete, Re-sequencing, Speciation, Hybridization, Selective sweeps, Secondary contact

17 RUNNING TItLE: Neurospora discreta speciation genomics 


\section{Abstract}

24 Delineating microbial populations, discovering ecologically relevant phenotypes, and identifying

25 migrants, hybrids or admixed individuals has long proved notoriously difficult, thereby limiting our

26 understanding the evolutionary forces at play during the diversification of microbial species. However,

27 recent advances in sequencing and computational methods have enabled an unbiased approach

28 whereby incipient species and the genetic correlates of speciation can be identified by examining

29 patterns of genomic variation within and between lineages. We present here a population genomic

30 study of a phylogenetic species in the Neurospora discreta species complex, based on the resequencing

31 of full genomes ( $\sim 37 \mathrm{Mb})$ for 52 fungal isolates from 9 sites in three continents. Population structure

32 analyses revealed two distinct lineages in Southeast Asia, three lineages in North America/Europe with a

33 broad longitudinal and latitudinal range, and limited admixture between lineages. Genome scans for

34 selective sweeps and comparisons of the genomic landscapes of diversity and recombination provided

35 no support for a role of selection at linked sites on genomic heterogeneity in levels of divergence

36 between lineages. However, demographic inference indicated that the observed genomic heterogeneity

37 in divergence was generated by varying rates of gene flow between lineages following a period of

38 isolation. Many putative cases of exchange of genetic material between phylogenetically divergent

39 fungal lineages have been discovered, and our work highlights the quantitative importance of genetic

40 exchanges between more closely related taxa to the evolution of fungal genomes. Our study also

41 supports the role of allopatric isolation as a driver of diversification in saprobic microbes. 


\section{INTRODUCTION}

45 The differentiation of lineages and the evolution of reproductive barriers between them are the

46 processes that create the tree of life. Understanding their underlying mechanisms is a major goal of

47 evolutionary biology. Progress has been made in the past decades on understanding the different

48 factors and traits causing lineage divergence and the evolution of reproductive isolation (Schluter 2000;

49 Coyne and Orr 2004; Nosil 2012; Stukenbrock 2013), or at the opposite, promoting hybridization or

50 genetic exchanges between long divergent lineages (Barton \& Hewitt 1981; Arnold 2004; Roper et al.

51 2011; Rius and Darling 2014). In addition, individual genomic features contributing to adaptive evolution

52 and reproductive isolation have been identified (Dettman et al. 2012; Nosil \& Schluter 2011; Presgraves

53 2007; Rieseberg \& Blackman 2010), and as the phylogenetic breadth and depth of taxa with sequenced

54 genomes has increased, many putative cases of exchange of genetic material between phylogenetically

55 divergent lineages have been discovered (Brand et al. 2013; Gladieux et al. 2014; Novo et al. 2009;

56 Racimo et al. 2015). Whole-genome data sets provide increased resolution that can reveal the sequence

57 and timing of speciation (lineage-splitting) events and the geographic context in which they occurred.

58 They also make it possible to address long-standing questions regarding the extent to which the

59 divergence of populations, or their maintenance, occur in the face of ongoing gene flow: How do

60 genomes diverge during speciation? How do they respond to secondary contact? How permeable are

61 they to post-divergence genetic exchanges?

62 Theory predicts that levels of genetic divergence across the genome will be more heterogeneous

63 in populations diverging in the presence of gene flow (sympatry or parapatry) than in geographically

64 isolated (allopatric) populations. Under the strictly allopatric model, gene flow between species ceases 
65 at every locus in the genome simultaneously so that the genealogical histories of all loci in the genome

66 are compatible with a single time of species divergence. But genomic data reveals that divergence is

67 often heterogeneous, and ancestral relationships among genomic features are not always congruent

68 with known phylogenetic history, suggesting that the timing and genealogical history of speciation might

69 be complex (Garrigan et al. 2012). Hybridization between diverging lineages may therefore create a

70 genomic mosaic of regions where interspecific gene flow has ceased at different times (the genic view of

71 speciation), due to the inability of genomic features directly involved in differential adaptation and/or

72 reproductive isolation to freely permeate species boundaries (Wu 2001). Several population-genetic

73 models that focus on selection at linked sites also predict greater differentiation in regions of low

74 recombination simply as a result of faster sorting of ancestral alleles even in the absence of gene flow

75 (Nachman \& Payseur 2012).

Filamentous fungi like the genus Neurospora are ideal systems for studying the nature and time

77 course of genetic changes underlying population divergence and species maintenance. Ecological

78 genomics of adaptation and speciation in Neurospora fungi is facilitated by their haplontic life-cycle and

79 their relatively small genomes with low proportions of repetitive DNA. Moreover, the genus contains

80 multiple recently diverged species, some of which coexist in sympatry or have large distributions

81 encompassing various environments. Thousands of wild strains representing broad taxon sampling have

82 been collected from all continents and are available from the Fungal Genetic Stock Center (Turner et al.

83 2001; Jacobson et al. 2004; Jacobson et al. 2006). Reproductive isolation can be measured using well-

84 tested protocols (Dettman et al. 2008; Dettman et al. 2003; Menkis et al. 2009; Turner et al. 2010), and

85 genomic tools and functional annotation resources are available to make connections between

86 genotype, phenotype and fitness (Davis \& Perkins 2002; Dunlap et al. 2007; McCluskey et al. 2010;

87 Ellison et al. 2011; Palma-Guerrero et al. 2013; Ellison et al. 2014). 
Previous population genomic studies provided insights into the environmental factors that may be

89 driving lineage divergence in Neurospora. They also evidenced divergence-with-gene-flow of Neurospora

90 populations and an important role of genomic novelties of trans-specific origin as sources of adaptive

91 change. Ellison et al. (2011) discovered two cryptic and recently diverged populations of N. crassa,

92 endemic to Louisiana and the Caribbean basin, and genome scans revealed at least two 'islands' of

93 extreme divergence between them, with growth-rate assays suggesting that divergence islands may be

94 the result of local adaption to latitudinally distinct environments, an hypothesis validated by gene

95 deletion. Analyses also suggested that the divergent haplotype observed at one of the islands of

96 divergence in the Louisiana population has been introgressed from another, unknown, Neurospora

97 species. Phylogenomics of mating type chromosomes also revealed the existence of large introgression

98 tracts in three phylogenetic lineages of the selfing species Neurospora tetrasperma, and analyses

99 showed that the introgression likely originated from two closely related outcrossing species (Strandberg

100 et al. 2010; Sun et al. 2012). To extend these findings, and to address their universality, we conducted a

101 population genomic study of another species of Neurospora: the Phylogenetic Species 4 of the $N$.

102 discreta species complex (=PS4). This species has the greatest latitudinal distribution of any Neurospora

103 species, from central Africa, through Europe and western North America, as far north as Alaska

104 (Dettman et al. 2006). The broad distribution of PS4, and the frequent coexistence of multiple $\mathrm{N}$.

105 discreta phylogenetic species in sympatry make them ideal systems to study the general processes

106 involved in the build up or maintenance of population differentiation. We used deep-coverage, and

107 genome-scale DNA sequence data from 52 genomes of PS4 and experimental measures of reproductive

108 isolation to address the following questions: What is the population structure? What is the genealogical

109 history of population subdivision? What are the genome-wide patterns of divergence? What is the

110 degree and genomic distribution of gene flow between related lineages? What is the degree of 
111 reproductive isolation between lineages caused by intrinsic prezygotic barriers and intrinsic early post-

112 zygotic barriers? What is the degree and genomic distribution of introgression from other lineages?

113 What are the genomic features harboring signatures of recent selective sweeps? Our results reveal

114 cryptic population structure within PS4, with two distinct lineages in Southeast Asia, three lineages in

115 North America/Europe with a broad longitudinal and latitudinal range. Demographic and clustering

116 analyses were also consistent with a model of secondary contact following a period of isolation, with

117 varying rates of introgression generating genomic heterogeneity in levels of divergence between

118 lineages.

119 Materials AND MEthods

120 Genomic data collection

121 Isolates from Cote d'Ivoire, Papua New Guinea, Thailand, Switzerland and Spain were provided by the

122 Fungal Genetic Stock Center. Isolates from CA, NM, and WA were obtained from DJ's personal

123 Neurospora collection, hosted in JT's lab. Isolates from AK were collected by JT and handled as described

124 in Jacobson et al. ( 2004).

125 Isolates were initially germinated on Vogel's minimal medium (Vogel 1956). After growth was

126 observed, cultures were transferred to a Petri dish containing Bird medium (Metzenberg 2004) overlaid

127 by cellophane and left to grow under constant light for $36-48 \mathrm{~h}$ before harvesting. Mycelial tissue was

128 placed in the -80 Celsius freezer for 30 minutes, and then incubated at $65^{\circ} \mathrm{C}$ for 45 minutes, inverting

129 occasionally, adding $0.5 \mathrm{~mL}$ of Zirconia/silica $0.5 \mathrm{~mm}$ beads and $500 \mu$ of lysis buffer with final

130 concentrations of $50 \mathrm{mM}$ Tris- $\mathrm{HCl}, 50 \mathrm{mM}$ EDTA, and 3\% SDS. The mycelium was ground twice for 30s in

131 a bead beater machine, and in between the material was re-suspended by shaking. After a wash with

$132500 \mu \mathrm{l}$ chloroform:isoamyl alcohol (24:1), samples were placed in the bead beater machine for another 
$13330 \mathrm{~s}$, and then centrifuged $(12,000 \times \mathrm{g}, 5 \mathrm{~min})$ to remove cellular debris. The aqueous phase was

134 collected and genomic DNA was extracted using the DNeasy Tissue Kit (Qiagen Inc., Valencia, CA).

135 Genomic libraries were generated manually or with Apollo 324 NGS Library Prep System (Wafergen

136 Biosystems), and samples were sequenced in Illumina HiSeq2000, 100bp paired-end with a 500bp insert

137 size at the QB3 Vincent J. Coates Genomics Sequencing Laboratory at the University of California,

138 Berkeley. The raw reads were deposited in the Sequence Read Archive and the accession numbers are

139 listed in Table S1 (Supporting Information).

$140 \quad$ Reads were filtered based on quality and all duplicates and reads with more than $10 \%$ Ns, with

141 at least one base with phred score smaller than 20, and with average phred score smaller than 30 were

142 discarded. All good quality paired and orphan reads were then aligned to the reference genome using

143 BOWTIE 2.0 (Langmead \& Salzberg 2012). We used --END-TO-END mode, which requires that the entire

144 read align from one end to the other, and preset option -SENSITIVE. We also used option --NO-DISCORDANT,

145 which specifies that pairs of reads that do not align with the expected relative mate orientation and with

146 the expected range of distances between mates are discarded. Alignments were sorted with SAMTOOLS

147 (Li et al. 2009), duplicates were removed with PICARD (http://picard.sourceforge.net) and reads with

148 mapping quality below 30 were removed with BAMTOoLs (Barnett et al. 2011). We used

149 RealignerTargetCreator and INDELREAligner within the GenOME ANALYSES Toolkit (McKenna et al. 2010) to

150 define intervals to target for local realignment, and to perform local realignment of reads around indels,

151 respectively, and UNIFIED GENOTYPER to call SNPS. We removed SNPs with quality <30, SNPs with mean

152 coverage below 5 and above 200 and sites with more than 33\% missing data using VCFTOOLS (Danecek et

153 al. 2011).

154 Polymorphism, recombination, divergence 
155 Preliminary phylogenetic analysis based on the full set of SNPs and isolates was carried out using the

156 programs DNADIST and neighbor from the PHYLIP 3.69 package. Polymorphism and divergence statistics

157 were computed using custom-made python scripts calling EGGLIB C++ libraries. $d x y$ was computed

158 following (Nei \& Li 1979) as the average number of pairwise differences between sequences from two

159 populations, excluding all comparisons between sequences within populations. $F_{S T}$ was calculated as $\left(\pi_{T}\right.$

$\left.160 \pi_{S}\right) / \pi_{T}$, where $\pi_{T}$ is the nucleotide diversity in the total sample and $\pi_{S}$ is the average nucleotide diversity

161 in each population (Nei 1982). Recombination rates were estimated for the 20 largest scaffolds using the

162 INTERVAL program in LDHAT version 2.2 (Auton \& McVean 2007). Singletons and sites with missing data

163 were excluded and the reversible-jump Markov-chain Monte Carlo scheme implemented in INTERVAL was

164 run for $5 \mathrm{e} 6$ iterations, with a block penalty of 10 , samples taken every 5000 iterations and a burnin

165 phase of 5e5. $Z$, the standardized Fay and Wu's $H$ was calculated using two outgroups (THA and PS4A)

166 and only sites where both outgroups had same alleles were included, to avoid biases caused by

167 homoplasy on the outgroup branch (Fay \& Wu 2000; Baudry and Depaulis 2003; Zeng et al. 2006). The

168 coefficient of linkage disequilibrium (r2) (Hill \& Robertson 1968) was computed using VCFTOoLs with

169 missing data excluded. The rate of decay in linkage disequilibrium with physical distance was

170 determined by averaging $\mathrm{r} 2$ for all pairs within a particular distance, with $1 \mathrm{~kb}$ increments.

171 Source-sink patterns within lineages

172 Allelic richness and private allele richness were calculated with ADZE v1.0 (Szpiech et al. 2008) for

173 subpopulations within lineages CA-WA and NM-WA based on haplotypes identified in 10kb windows.

174 Only SNPs without missing data were included. ADZE implements a rarefaction procedure for counting

175 alleles while adjusting for differences in sample size across subpopulations. Calculations were made

176 using a standardized sample size corresponding to the smallest number of observations per population

$177 \quad(n=3)$. 
179 We used RAxML (Stamatakis 2014) to infer maximum likelihood genealogies based on the full set of

180 SNPs or on $10 \mathrm{~kb}$ genomic windows. In scans of topological variation along the genome, only branches

181 with bootstrap support above $70 \%$ were considered. We used 100 bootstrap replicates.

\section{Admixture}

183 The three-population test is a test for treeness in three population trees including a focal group $\mathrm{X}$ and

184 two other groups $\mathrm{Y}$ and $\mathrm{W}$. If group $\mathrm{X}$ is related to groups $\mathrm{Y}$ and $\mathrm{W}$ by a simple tree (i.e., without

185 admixture), the $f 3$ statistic should be positive. If group $X$ results from admixture of groups related to $Y$

186 and $W, f 3$ can be negative, and thus a significantly negative value of $f_{3}$ provides an unambiguous signal

187 of admixture (Reich et al. 2009). $f 3$ tests were carried out using TREEMıx (Pickrell \& Pritchard 2012) with

188 standard errors computed in blocks of 100 SNPs.

\section{Demographic history inference}

190 We used the PS6 and THA genome sequences as outgroups to infer ancestral alleles and restricted our

191 dataset to include only those SNPs for which both outgroups shared the same allele, in order to limit

192 misidentification of ancestral alleles, which can bias demographic inference. Twenty-three divergence

193 models were considered (Table S2, Supporting Information), and for each model we ran the program

194 multiple times with different starting points to ensure convergence. We used the simplex method

195 implemented in dadi.Inference.optimize_log_fmin for numerical optimization, as we found that the

196 BFGS-based routines often failed to arrive at a good estimate starting from a random point in parameter

197 space. We assessed the model's goodness-of-fit by maximizing the model likelihood and visual

198 inspection of the residuals between the site frequency spectra generated by the inferred model and the 
199 real data. We used 100 nonparametric bootstraps to estimate parameters uncertainties, using 100

200 pseudo-observed datasets with equal numbers of loci sampled with replacement from the original data 201 set.

202 Selective sweep analysis

203 To identify genomic features that have potentially swept through populations we applied the genome

204 scan method implemented in the program H-SCAN, which aims to detect signatures of selective sweeps

205 by measuring the average length of pairwise homozygosity tracts ( $H$ statistic) in a population sample.

206 The $H$ statistic was computed for all variable sites and all lineages.

\section{Mating success}

208 The mating type of each isolate was determined in silico, using BLASTN of the mat $A$ (Staben \& Yanofsky

209 1990) and mat a (Glass et al. 1990) sequences on genomic sequences assembled using SOAPDENOVO.

210 Crosses were made in $13 \times 100-\mathrm{mm}$ tubes containing slanted Westergaard synthetic crossing medium.

211 We used filter paper as a carbon source to reduce conidiation (i.e., production of mitotic spores) and

212 because it is an effective substrate for crossing Neurospora (Perkins \& Turner 1988; Turner et al. 2001).

213 Filter paper was cut into strips and inserted into the tubed medium prior to the autoclaving and slanting

214 processes. Strains were incubated for 4 days at $25^{\circ} \mathrm{C}$ to allow for the development of receptive

215 protoperithecia (producing specialized hyphae, trichogynes, which are the female gametes), and

216 fertilization was performed by transferring mitotic spores (i.e, male gametes) using a platinum loop.

217 After an additional 14 days of incubation, the reproductive success was scored on a scale with five rating

218 categories under a dissecting microscope (Figure 5). The five categories correspond to different stages in

219 reproductive development, from the development of perithecia, to the formation of black ascospores

220 (Jacobson 1995; Dettman et al. 2003). Unlike other species of Neurospora, N. discreta ascospores are 
221 not necessarily ejected from perithecia and can simply be oozing out. Hence, we determined the

222 proportion of black ascospores visually after crushing perithecia in water between microscope slide and 223 a cover slip.

\section{RESULTS}

\section{Genome alignment and SNP calling}

226 We present a population and comparative genomics analysis of $N$. discreta Phylogenetic Species 4 (PS4)

227 evolution based on the resequencing of 52 genomes (Table 1). We used Illumina short-read technology 228 to generate genome-wide sequences from 43 isolates of the PS4 group B (= PS4B): 8 from Alaska (AK),

22915 from California (CA), 8 from New Mexico (NM), 8 from Washington (WA), and 3 non-North American 230 isolates [2 from Europe (EU), one from Thailand (THA)]. We also used 9 isolates of PS4 group A from

231 Papua New-Guinea (=PS4A), and as the outgroup one isolate of $N$. discreta PS6 from Côte d'Ivoire (CIV).

232 Reads were mapped to the $37.3 \mathrm{Mb}$ N. discreta PS4 reference genome (hosted on FungiDB

233 http://fungidb.org; isolate FGSC 8579, collected in Belen, NM), yielding average alignment depths of

$23412.4 \times$ to $108.6 \times$ and covering $77.8 \%$ to $98.9 \%$ of the reference sequence (Table 1 ; Table S1, Supporting

235 Information). After a preliminary phylogenetic analysis, the dataset was clone-corrected by keeping a

236 single representative of nearly identical genotypes (i.e., where genetic distance between genotypes is

237 below 0.1; F84 distance, DNADIST program, PHYLIP package; Table S1; Figure S1, Supporting Information).

238 Analyses were based on a final set of 42 genomes representing 1,762,444 high-quality SNPs of which

239628,558 had no missing data. We note that the role of conidia for Neurospora dispersal in nature has

240 long been suspected (Pandit and Maheshwari 1996; Turner et al. 2001) and interestingly our finding of

241 clonal genotypes provides the first indirect evidence that conidia can start new colonies and contribute

242 to spread the fungus. 
244 To assess the total evidence for population subdivision and the genealogical relationships among

245 samples, the complete set of SNPs was used to infer a maximum likelihood phylogenetic tree. In the best

246 fitting tree, rooted by the PS6 isolate from Côte d'Ivoire, the PS4B isolate from Thailand branched in

247 between PS4A and other PS4B isolates (Figure 1). A total of 1,189,625 SNPs were specific to the

248 combination of North American/European PS4B and PS4A sequences, of which 15,647 (1.3\%) were

249 shared and 355,484 fixed (29.9\%) between the two groups. A total of 516,729 SNPs were segregating in

250 the North American/European dataset alone.

251 Within PS4B sequences from Europe and North America, we observed three distinct lineages,

252 hereafter referred to as the AK-EU, CA-WA and NM-WA lineages (Figure 1). The CA-WA lineage was

253 basal to the group formed by the AK-EU and NM-WA lineages and comparisons between pairs of

254 lineages showed that the vast majority of variable sites corresponded to fixed differences between

255 lineages, or polymorphisms that were exclusive to each lineage (Table 2). Interestingly, isolates from the

256 two lineages found in Washington state (WA) were coexisting in sympatry within the same, small

257 campground at Lake Chelan.

258 We also analyzed population subdivision and admixture within the North American/European

259 group of samples using the model-based clustering algorithm implemented in FRAPPE. Only runs with $K=2$

260 and $K=3$ populations converged consistently towards the same likelihood values and towards the same

261 clustering solution, and the greatest increase in likelihood among runs was observed from $K=2$ to $K=3$.

262 Together, these results suggest that the model with $K=3$ populations captures most of the population

263 subdivision signal existing in the North American/European clade (Figure S2, Supporting Information),

264 and the three identified clusters matched the lineages identified using maximum likelihood phylogenetic 
265 inference (Figure 1). Admixture among clusters was only observed in Alaska where one individual of the

266 Alaskan subpopulation had substantial ancestry in the NM-WA lineage (membership coefficient in NM-

267 WA: 12.04\%). A genome scan revealed that putatively introgressed segments could be detected and

268 were widespread in the genome of the admixed Alaskan isolate (Figure S3, Supporting Information). This

269 is consistent with relatively recent hybridization and backcrossing between the two lineages, although

270 the isolates from within the NM-WA lineage had not been detected in Alaska.

271 Turning to the two, sympatric subpopulations coexisting in Washington, using the three-

272 population test, a formal test of admixture based on patterns of allele frequency correlation that can

273 detect gene flow events occurring over hundreds of past generations (Reich et al. 2009; Patterson et al.

274 2012), we found no support for an admixed ancestry in Washington subpopulations either for the CA-

275 WA lineage (sources: $C A, N M ; f 3=0.00091 ; Z$-score=0.76773) or the NM-WA lineage (sources: CA,NM;

$276 f 3=0.00155 ;$ Z-score=3.13093).

The geographic range of the three lineages could be consistent with recolonization from either

278 southern or northern glacial refugia. To investigate source-sink patterns, and given the few Washington

279 isolates in either the CA-WA or NM-WA lineages ( $n=3$ for each Washington component), we used an

280 approach based on the computation of allele richness and private allele richness rather than the more

281 familiar coalescent-based approach. The allele richness approach rests on the assumption that recently

282 founded populations retain only a subset of the variability existing in parental populations. We

283 estimated allele and private allele richness in 10kb windows (i.e., 10kb haplotypes defined the different

284 'alleles'), using a rarefaction procedure designed to avoid biases due to differences in sample size

285 between populations from each pair (Szpiech et al. 2008). We found higher levels of allele richness (AR)

286 and private allele richness $(P A R)$ in the WA subpopulations for both lineages, suggestive of histories of

287 independent southward migrations from WA to CA and from WA to NM (Table 3). 
289 Summaries of variation are listed in Table 4. Variability, estimated by the nucleotide diversity $(\pi)$, the

290 proportion of polymorphic sites per base $\left(\vartheta_{w}\right)$, number of haplotypes $(K)$ and haplotype diversity $(H e)$

291 averaged across $10 \mathrm{~kb}$ windows, was higher in PS4A $\left(\pi=0.0032 / \mathrm{bp} ; \vartheta_{w}=0.0036 / \mathrm{bp} ; \mathrm{He}=0.923 ; K=7.993\right)$,

292 intermediate in CA-WA $\left(\pi=0.0021 / \mathrm{bp} ; \vartheta_{w}=0.0020 / \mathrm{bp} ; \mathrm{He}=0.880 ; K=7.910\right)$, and lower in AK-EU

$293\left(\pi=0.0009 / \mathrm{bp} ; \vartheta_{w}=0.0009 / \mathrm{bp} ; H e=0.773 ; K=5.788\right)$ and NM-WA $\left(\pi=0.0007 / \mathrm{bp} ; \vartheta_{w}=0.0007 / \mathrm{bp}\right.$;

$294 H e=0.823 ; K=6.094)$. The recombination rate was on the order of 10 Morgans $/ k b$ for all lineages,

295 compared to 1 to 3 Morgans/kb for populations of Saccharomyces cerevisae (Tsai et al. 2008) (Table 4).

296 Linkage disequilibrium decayed to half its maximum value in less than $1 \mathrm{~kb}$ to $3 \mathrm{~kb}$ (Table 4), compared to

297 0.78kb in N. crassa (Ellison et al. 2011) and 3kb in S. cerevisae (Liti et al. 2009). Computations based on

298 local subpopulations (i.e., the AK, CA, NM and two WA samples) instead of lineages that had been

299 identified using clustering algorithms gave the same pattern (data not shown).

We observed a large number of high-frequency derived mutations in all lineages, leading to U-

301 shaped (i.e., non-monotonic) derived Site-Frequency Spectra (SFS) (Figure 2). Model fitting using the

302 PYTHON package DADI (Gutenkunst et al. 2009) indicated that observed SFS were significantly different

303 from those expected under the standard neutral model $\left(\chi^{2}>300 ; P<0.001\right)$. We observed a systematic

304 excess of high-frequency derived variants, and nearly all lineages also displayed an excess of low

305 frequency variants compared to neutral expectations (Figure 2). The observed excess of high-frequency

306 derived mutations was not due to misidentification of ancestral states, as we restricted the dataset to

307 include only those SNPs for which the two outgroups had same alleles to avoid biases caused by

308 homoplasy on the outgroup branches. The fact that the non-synonymous SFS was more skewed toward

309 low-frequency variants than the synonymous SFS in all lineages but CA-WA suggests that negative 
310 selection, which maintains deleterious mutations at low frequencies, contributes to the excess of rare

311 polymorphisms, possibly in combination with recent population expansion. Moreover, the fact that the

312 synonymous SFS was more skewed toward high-frequency variants than the non-synonymous SFS

313 suggests little role for genetic draft (Gillespie 2001; Maynard-Smith et al. 1974) in the large proportions

314 of high frequency derived polymorphisms. The fitting of multiple-populations models indicated that this

315 latter category of variants may be explained by migration among populations (see below).

316 Genealogical discordance across the genome

317 Unlike the simple allopatric model which predicts a single split time for all genomic regions, most non-

318 allopatric speciation models predict discordant genealogical histories among loci (Garrigan et al. 2012;

319 Martin et al. 2013). To characterize the genome-wide distribution of genealogical histories resulting

320 from divergence within PS4B, we inferred maximum likelihood genealogies for $10 \mathrm{~kb}$ windows using PS6

321 as an outgroup. Of 3797 windows, $41.8 \%$ presented 'resolved' genealogies, that is, with the outgroup

322 specifying one of the North American/European lineages as basal and the other two lineages forming a

323 monophyletic, sister group. CA-WA was identified as the basal group in $17.5 \%$ of genomic windows

324 while discordant topologies with either the NM-WA or the AK-EU lineages as basal groups were found in

325 trees for $12.7 \%$ and $11.7 \%$ of genomic windows, respectively. The most supported phylogeny of North

326 American/European lineages based on the full set of SNPs ((AK-EU,NM-WA),CA-WA) was retrieved in

$32712.7 \%$ of $10 \mathrm{~kb}$ windows, and it was a subset of the $17.5 \%$ genealogies with an internal branch between

328 CA-WA and the remaining AK-EU and NM-WA isolates, regardless of the separation between these latter 329 two lineages.

330 Inter-lineage gene flow 
331 The finding of admixture between AK-EU and NM-WA suggested that the different lineages may also

332 have been connected by gene flow in the past. To test whether the observed widespread phylogenomic

333 discordance across PS4B genomes was due to post-divergence gene flow, we inferred a complete

334 demographic model using the DADI package (Gutenkunst et al. 2009), which infers demographic

335 parameters based on a diffusion approximation to the site frequency spectrum. We used a sequential

336 approach, as the method cannot handle more than three population samples. The compared models

337 included scenarios of strict isolation, continuous post-divergence gene flow, ancient migration and

338 secondary contact, assuming or not heterogeneous migration rates across the genome. Given a model,

339 we used DADI to generate an expected site frequency spectrum, to maximize the similarity between the

340 expected and observed site frequency spectra over the parameter values space, and to evaluate fit by

341 computing a composite-likelihood. We first inferred the demographic history of lineages PS4A and PS4B-

342 US-EU, the latter corresponding to the pooling of the three North-American/European lineages. We

343 started from a simple bifurcating model with constant population size and no migration, and we

344 progressively increased model complexity by adding parameters. The most supported model included a

345 size change in the ancestral population, simultaneous size changes in descendant lineages, and relatively

346 recent gene flow following secondary contact between them (Figure 3; Supplementary Table 2).

347 Examination of residuals indicated that the resulting model reproduced the observed spectra well for

348 intermediate-frequency variants, but we also found an excess of high frequency alleles and a deficit of

349 low frequency alleles in our data compared with what is predicted by the model (Figure 2; Figure S4,

350 Supporting Information). In order to convert parameter estimates to physical units, we estimated the

351 ancestral population size $\left(N_{A B 1}\right)$. We used the population mutation rate $\vartheta=4 N_{A B 1} \mu L$, where $\mu$ was

352 assumed to be approximately 1 e -9 per generation (Lynch 2010), and $L$ is the genome size (ca. 37Mb).

353 This ancestral population size was then used to transform time estimates from DADI (in units of $2 N_{A B 1}$ ) 
354 into actual generations. The estimated divergence time was $1.8 \mathrm{e} 6$ generations ago (i.e., $2.9 \mathrm{e} 5+3.6+$

355 1.5e6; Figure 3; Table 5). Parameter estimates indicated relatively recent and severe population

356 shrinkage in PS4B-US-EU (from 8.3e9 to 9.2e4 individuals) and ancient, strong population increase in

357 PS4A (from 2.1e5 to $1.6 \mathrm{e} 8$ individuals), with both size changes estimated to have occurred 2.9e5

358 generations ago. We also inferred an ancient population expansion in the ancestral population (ca. 1000

359 fold increase, $1.5 \mathrm{e} 6$ generations prior to lineage splitting). Secondary contact and gene flow was

360 estimated to have started at approximately the same time as population size changes. The migration

361 rate was substantial into PS4A (6.4e-8 genetic replacement per generation) and nearly zero into PS4B-

362 US-EU (2.1e-42 genetic replacement per generation); the corresponding effective number of migrants

363 per generation was on the order of $1 \mathrm{e}-1$ and $1 \mathrm{e}-36$, respectively.

364 We then inferred the demographic history of lineages within PS4B-US-EU. We started from the

365 inferred total evidence maximum-likelihood tree as the true population phylogeny, and we progressively

366 increased model complexity by adding parameters. The best-supported model included a strong

367 demographic bottleneck in the ancestral population prior to divergence and relatively recent

368 heterogeneous migration among lineages following secondary contact (Figure 3; Table S2, Supporting

369 Information). Overall fit was also good, with the resulting model reproducing the observed spectra well,

370 as seen in residuals (Figure 2; Figure S4, Supporting Information). Parameter estimates indicated a

$371 \sim 1000$ fold decline in the global joint ancestral population, a small effective size and short lifespan of the

372 population ancestral to lineages AK-EU and NM-WA, and effective population sizes on the order of 1e4

373 to 1 e5 for derived lineages (Table 5; Figure 3). The three lineages diverged ca. 4.4e5 generations ago,

374 and gene flow following secondary contact began 7.4e4 generations ago. Most of the genome (85.1\%)

375 experienced migration rates on the order of 1e-7 genetic replacement per generation (corresponding

376 effective number of migrants per generation on the order of $1 \mathrm{e}-3$ to $1 \mathrm{e}-2)$, except for migration into 
377 NM-WA from AK-EU, which was zero. A smaller fraction of the genome (14.9\%) experienced migration

378 rates that were 100 times higher from NM-WA into CA-WA, 10 times higher from NM-WA into AK-EU,

379 similar from CA-WA into AK-EU, 10 times smaller from CA-WA into NM-WA and from AK-EU into CA-

380 WA, and on the order of 1e-8 genetic replacement per generation from AK-EU into NM-WA

381 (dramatically higher migration than the $1 \mathrm{e}-13$ estimated for the other $85 \%$ of the genome). Gene flow

382 along the internode carrying the AK-EU and NM-WA lineages could not be estimated (data not shown)

383 and the corresponding parameters were therefore not included in the models compared.

\section{Genome-wide patterns of divergence}

385 To measure the extent of isolation between lineages and subpopulations at different levels of

386 geographic separation, while accounting for genomic heterogeneity in levels of divergence, we

387 characterized patterns of genetic divergence across the genome using measures of relative divergence

388 (Fst, also known as a measure of 'differentiation') and absolute divergence (dxy) (Figure 4). The

389 divergence metrics were computed at the scale of non-overlapping 10kb windows in order to increase

390 precision by minimizing noise from individual site-based estimates. Overall, levels of divergence were

391 consistent with the phylogenetic relationships among taxa: average divergence was greater between

392 PS4A and PS4B than between lineages within PS4B, and greater between PS4B lineages than between

393 subpopulations within them (Table 6).

Theory predicts that variation in genome-wide levels of divergence as a result of the mode (with

395 or without gene flow) and stage of separation between populations. Thus, levels of divergence are

396 expected to be low and homogenous during early stages of speciation when gene flow is high, and rise

397 in level and heterogeneity (due to unevenness in susceptibility to introgression) as the speciation

398 process proceeds, and reach a high and more homogenous level after the speciation process is 
400 we found that the coefficient of variation followed this trend, with lowest levels of heterogeneity in

401 divergence in comparisons between PS4A and PS4B, and highest levels in comparisons between

402 subpopulations from within the same lineage. Complicating the picture is the hypothesis that

403 heterogeneity in levels of divergence is affected by the existence and strength of linked selection and by

404 genomic variation in rates of recombination such that positive or negative selection are expected to

405 increase levels of relative divergence in regions of low recombination (Nachman \& Payseur 2012). We

406 found no significant, positive correlation between local recombination rate and nucleotide diversity (not

407 shown), suggesting no strong impact of background selection and/or hitchhiking (Hill-Robertson effects)

408 on patterns of diversity, and thus no strong contribution of selection at linked sites to heterogeneity in

409 levels of relative differentiation.

410 Theory also predicts that the variation in levels of divergence across the genome is greater in

411 populations diverging in the presence of gene flow (sympatry or parapatry) than in geographically

412 isolated populations. Levels of divergence between sympatric populations of the CA-WA and NM-WA

413 lineages were broadly similar in mean and variation to those between their allopatric counterparts

414 (Table 6). This result suggests that gene flow was not higher between sympatric populations relative to

415 allopatric ones, consistent with the lack of evidence for recent hybridization between these sympatric

416 subpopulations in analyses of admixture (Figure 1).

\section{Genome scan for selective sweeps}

418 In a selective sweep, rare adaptive variants and linked variation are driven to high population

419 frequencies by positive selection, while non-adaptive alleles are removed from the population. Selective 420 sweeps are classified as 'hard' sweeps, where only a single adaptive allele sweeps through the 
421 population, and 'soft' sweeps, where multiple adaptive alleles sweep at the same time through the

422 same population or through different populations of a genetically structured species (Messer \& Petrov

423 2013). Hard and soft sweeps are both expected to increase linkage disequilibrium over neutral

424 expectations and generate unusually long tracts of homozygosity around the adaptive site.

425 To identify genomic features that have potentially swept through populations we applied the

426 genome scan method implemented in the program H-SCAN, which aims to detect signatures of selective

427 sweeps by measuring the average length of pairwise homozygosity tracts ( $H$ statistic) in a population

428 sample. We measured the average pairwise homozygosity tract length (in bp) extending in both

429 directions from each SNP. We weighed the tract lengths by the number of SNPs in each pairwise

430 homozygous region to account for variable SNP density. The $H$ statistic was computed for all variable

431 sites and all lineages (Figure S5, Supporting Information). However, the highest $H$ values all

432 corresponded to relatively small regions of elevated pairwise homozygosity (8400bp on average),

433 indicating a lack of recent detectable selective sweeps in PS4 lineages (Figure S5; Table S3, Supporting

434 Information).

435 Experimental measures of reproductive success

436 The lack of admixture between subpopulations coexisting in sympatry at the Washington site suggested

437 the existence of strong reproductive isolation barriers between the CA-WA and NM-WA lineages. To test

438 this hypothesis and more generally to gain insight into the strength and nature of reproductive isolation

439 among subpopulations and lineages, we experimentally measured several components of mating

440 success of crosses between 29 of the 31 North American and European isolates of PS4B. We also crossed

441 isolates to the original testers strain of N. discreta sensu stricto (Mat A P581 and Mat a 8127), in an

442 effort to assign them to a biological species. Mating types were determined computationally and we 
443 measured mating success for $16 \times 14=240$ crosses. Each isolate was used as both a female or a male, so

444 that each cross consisted in two matings, and reproductive success was reported as the most successful

445 of the two matings (Figure 5).

446 The vast majority of all attempted crosses were compatible (23 category 5, 208 category 6). Five

447 out of eight incompatible interactions were observed between isolates from the same lineage, two in

448 crosses with one of the tester strains, and only one unsuccessful cross involved isolates from different

449 lineages (Figure 5). Several isolates tended to be sterile when acting as the perithecial parent, but were

450 competent when functioning as the fertilizing parents, confirming previous observations that

451 reproductive success is influenced by the interaction of both parental genotypes (Dettman et al. 2003).

452 In general, mat A isolates showed a female sterility pattern, but not in all intra- or inter-group matings,

453 as illustrated by the success of crosses between CA-WA isolates or crosses between AK-EU mat A and

454 CA-WA mat a, suggesting that interactions between parental genotypes are modulated by the

455 geographic origin of the parents.

\section{Discussion}

457 To investigate the evolutionary factors responsible for the diversification of fungal populations and

458 species in the presence of gene flow, we used population genomics of 52 resequenced isolates from 9

459 sites in 3 continents combined with experimental measures of reproductive isolation to study

460 population structure in a fungus with an exceptionally broad latitudinal and longitudinal range, the fire-

461 associated fungus Neurospora discreta, a relative of $N$. crassa. Clustering analyses identified several

462 long-diverged lineages within the phylogenetic species PS4, and analyses of the genomic landscape of

463 divergence revealed extensive heterogeneity in levels of genomic divergence between lineages.

464 Haplotype-based statistics and analyses of genomic patterns of variation revealed no signature of 
465 selective sweeps and linked selection, indicating that genomic heterogeneity in divergence is not simply

466 an incidental consequence of selection at linked sites reducing within lineage variation relative to

467 divergence between populations. Modeling a number of demographic histories revealed that genomic

468 heterogeneity was most likely caused by differential gene flow following secondary contact between

469 allopatric lineages.

470 Biogeography and Neurospora's life cycle

471 Previous studies revealed that PS4 is the most broadly distributed species of Neurospora (Dettman et al.

472 2003; Jacobson et al. 2004; Jacobson et al. 2006). Our study demonstrates clear and deep population

473 structure within PS4, revealing the power of genomic sequencing to reveal cryptic diversity that may be

474 missed by more traditional methods of species recognition, such as phylogenetic investigations of

475 diversity (Taylor et al. 2000; Dettman et al. 2003). Model-based inferences of past demographic history

476 indicated that PNG and North American/European lineages diverged 3.6e6 generations ago, and

477 lineages within North America/Europe about 4.4e5 generations ago. Relating these estimates with past

478 geologic or climatic events requires an estimate of generation time, which is a perennial question in

479 fungi. Extensive examinations of Neurospora colonies at burn sites in North America led to only a single

480 observation of fruiting bodies beneath the bark of woody species (Jacobson et al. 2004). This suggests

481 that sexual reproduction in Neurospora may be induced by wildfires, and thus that the generation time

482 of Neurospora may correspond to the fire returns intervals. Previous work estimated a frequency of

483 wildfires ranging from 20-25 years for shrubs and grasslands to 300 years for pine forest in the

484 Yellowstone National Park (Houston 1973; Romme and Despain 1989), and ca. 100 years for the boreal

485 forest of Quebec (Lesieur et al. 2002). These estimates, however, are based on tree fire-scars

486 chronologies, which do not go further than one millenium, and it is not certain that fire returns intervals 
remained constant through time. Assuming 100 years as a gross estimate of the generation time of

488 Neurospora, the North-American/European and Papua-New-Guinean lineages of PS4 would have

489 diverged about 400 million years ago, which corresponds to the age of Ascomycota/Basidiomycota

490 divergence (Berbee \& Taylor 2006), and is much older than the divergence between $N$. discreta sensu

491 lato and other Neurospora heterothallic species estimated from phylogenies (ca. 7 My; Corcoran et al.

492 2014). This conflict suggests that fires were more frequent than estimated and/or that sexual cycles in

493 Neurospora are not necessarily synchronized with wildfires. Assuming, very conservatively, that the

494 divergence between PS4A and PS4B is the age of $N$. discreta sensu lato (7 My), our per-generation

495 estimate of $3.6 \mathrm{e} 6$ generations would actually imply a generation time of ca. 2 years, which is much

496 shorter than the estimated frequency of wildfires in North America.

497 Field observations (Jacobson et al. 2004) and experimental inoculations of pine seedlings (Kuo et

498 al. 2014) suggest that species of Neurospora may be living as an endophyte. An endophytic lifestyle

499 implies strong dependence of the migration pathway of Neurospora species on their hosts, although the

500 lack of host specificity make them less dependent on changes in the geographic range of a particular

501 species than on movements of the whole plant community. The oldest split detected within our dataset,

502 between North-American and Asian lineages of PS4, may be related to the dispersal and subsequent

503 isolation of plant species between landmasses across what is now the Bering land bridge, which existed

504 from the Miocene (23-5 Mya) toward the end of the Pliocene (3.5 Mya) (Wen 1999).

505 The geographical extent of our sampling effort limits detailed inferences on the phylogeography

506 of North-American lineages, but a number of hypotheses can nonetheless be proposed. Divergence

507 within North American PS4B may have been driven by climate oscillations of the Pleistocene (2.6Mya-

508 11kya), followed by secondary contact and limited hybridization along the Pacific Northwest, which is an

509 important suture zone between multiple Northward and Southward postglacial expansion routes 
510 (Swenson \& Howard 2005). The presence of the same lineage in Alaska and Europe may result from

511 long-distance dispersal, possibly via the transportation of logs or other woody material carrying the

512 fungus. This hypothesis is consistent with the lack of divergence between Alaskan and European samples

513 in the phylogeny inferred from the full set of SNPs. Such anomalies in the distribution of Neurospora

514 lineages have already been reported in N. crassa (Radic et al. 2012) and N. tetrasperma (Corcoran et al.

515 2014), and molecular data provided evidence that even fungal species without long-distant colonization

516 abilities can successfully establish themselves on new continents (Fontaine et al. 2013; Gladieux et al.

517 2015).

518 Reproductive barriers between sympatric lineages

519 Biological species in Neurospora have historically been delineated by measuring the proportion of black

520 ascospores in laboratory crosses (Perkins et al. 1976; Jacobson et al. 2004). When using this broad

521 criterion, we find that all investigated strains belong to a single biological species, in accordance with

522 their membership in the same phylogenetic species N. discreta PS4. However, despite compatibility in

523 vitro and the relatively recent divergence among North-America/European lineages, our resequencing

524 data provided no evidence for admixture between lineages CA-WA and NM-WA coexisting at

525 Washington sampling site. This lack of evidence suggests reproductive isolation between these lineages,

526 although the non-zero estimates of gene flow between them indicates that reproductive isolation

527 became effectively complete only recently, or is not complete between all populations of the two

528 lineages. As is always the case, it is not known if increased sampling might also reveal admixed

529 individuals that were not included in our study.

530 The widespread compatibility of crosses attempted in our in vitro study is not contradictory with

531 the fact that lineages mostly maintain cohesion, in particular in sympatry (Gladieux et al. 2011; Taylor et 
532 al. 2015; Taylor et al. 2000). The observed interfertility simply indicates that the relative reproductive

533 isolation between lineages is not caused by the intrinsic reproductive barriers which have been

534 evaluated in vitro, such as intrinsic premating barriers (i.e., assortative mating by mate choice), intrinsic

535 postmating prezygotic barriers (i.e., gametic incompatibility) or a form of intrinsic early postzygotic

536 isolation (i.e., zygotic mortality). Instead, the relative reproductive isolation between lineages appears to

537 be caused by one or several of the following extrinsic barriers (Table 1 in Nosil et al. 2005): temporal

538 isolation (i.e., different mating times), habitat isolation (i.e., different mating sites), ecological barriers

539 (i.e., immigrant inviability, selection against hybrids), or intrinsic late postzygotic barriers (i.e., hybrid

540 sterility), or another form of intrinsic early postzygotic isolation (i.e., hybrid inviability).

541 Among possible barriers to gene flow, the contribution of intrinsic barriers could be measured

542 experimentally, by germinating ascospores to estimate hybrid viability, and by using the resulting

543 mycelia to assess the self-fertility of hybrids. In N. tetrasperma, incorporating characters such as viability

544 and fertility of offspring resulted in a better congruence between biological species delineation and

545 actual patterns of historical gene flow (i.e., phylogenetic species recognition; Menkis et al. 2009).

546 Quantifying the role of extrinsic barriers to gene flow is more challenging in organisms that are difficult

547 to study in nature like Neurospora, but ecological barriers can nonetheless be investigated, indirectly,

548 using a reverse ecology approach (Ellison et al. 2011; Branco et al. 2015). In the case of North-

549 American/European lineages of PS4, overall levels of genomic divergence were too high, despite

550 substantial levels of gene flow, to allow the identification of candidate genomic features involved in the

551 local adaptation using genome scans. However, the finding of a late generation hybrid between NM-WA

552 and AK-EU lineages in Alaska suggest that reproductive isolation is not complete among all lineages, and

553 there might be opportunities to identify hybrid swarms that would allow fine mapping of genomic 
554 regions resisting to gene flow because of endogenous (i.e., incompatibilities) or exogenous (i.e.,

555 divergent ecological selection) reproductive barriers.

556 A comprehensive survey of the literature revealed a general lack of intrinsic premating isolation

557 between sympatric species of pathogenic Ascomycetes (Le Gac \& Giraud 2008) which is reminiscent of

558 the case of sympatric lineages of Neurospora (Dettman et al. 2003; Menkis et al. 2009; this study). The

559 authors proposed that the lack of intrinsic premating barriers between pathogenic Ascomycetes results

560 from a peculiarity of their life cycle, with mating in these fungi taking place within the host, so that

561 mating only occur between individuals adapted to the same host and hence host specificity

562 pleiotropically controls assortative mating (Le Gac \& Giraud 2008; Giraud et al. 2010; Gladieux et al.

563 2011). However, Neurospora species are not pathogens, but more likely endophytes, and albeit probably

564 mating within their hosts, they lack host specificity so it is unlikely that this form of habitat specialization

565 contributes to reproductive isolation. Another hypothesis is that the general lack of intrinsic premating

566 barriers between Neurospora species results from other factors, such as a the existence of constraints

567 on the evolution of such barriers (Turner et al. 2010; Turner et al. 2011).

568 Ongoing admixture and post-divergence gene flow

569 We inferred relatively high migration rates following secondary contact between North-

570 American/European lineages (median of effective migration rates on the order of 1e-1), and between

571 PS4B-US-EU and PS4A (on the order of 1e-1, unidirectional into PS4A), providing evidence that lineages

572 were connected by gene flow in the recent past, and therefore that their distributions have been

573 overlapping to some extent. The geographic distance between Papua New Guinea and North America

574 undoubtedly surpasses intrinsic dispersal abilities of Neurospora $(>10,000 \mathrm{~km})$ and our time estimate for

575 secondary contact (ca. $3 \mathrm{e} 5 \mathrm{ybp}$ ) greatly exceeds the first long-distance human travels across the Pacific 
576 Ocean (Jobling et al. 2004). One possibility is that detected gene flow might in fact be caused by

577 introgression by an unknown species of intermediate divergence between the two lineages, giving the

578 false impression that genetic exchanges occurred between the two allopatric lineages. Endemicity of this

579 putative species in Papua New Guinea may account for the observed asymmetry in migration patterns

580 (unidirectional migration into PS4A).

In North-America/Europe, the different lineages may have diverged following repeated periods of

582 isolation in separate glacial refugia, interspersed with periods of secondary contact potentially

583 permitting gene flow that only began relatively recently, as suggested by the finding of a late generation

584 hybrid in Alaska indicative of ongoing admixture. Patterns of allele richness and private allele richness in

585 populations from CA, WA and NM were consistent with histories of southward migrations, but a larger

586 sampling is required to conclude about the phylogeography of PS4 in North America. Patterns of genetic

587 variation consistent with allopatric isolation and subsequent secondary contact were also detected

588 North American lineages within the N. tetrasperma species complex by Corcoran et al. (2014).

Using a secondary contact demographic model with heterogeneous gene flow across the genome,

590 we were able to show that a small fraction of the genome experienced higher migration rates between

591 certain pairs of lineages. The indirect effects of selection presumably explain this heterogeneity in

592 introgression rates among genomic regions, with limited gene flow in the neighborhood of barrier loci

593 (genetic incompatibilities or genomic features under divergent ecological selection), maximal gene flow

594 at adaptively introgressed regions and basal gene flow in regions not affected by selection (Roux et al.

595 2013). It is important to note that the failure to account for genomic heterogeneity in introgression

596 rates would have provided support to an inappropriate demographic model of divergence, biasing

597 conclusions about the effects of natural selection on effective migration rates (Roux et al. 2014).

\section{Concluding remarks}


Population genomics offers great potential for enhanced understanding of the population biology and

600 evolution of fungi. Here, we reported on the population structure and demographic history of Asian,

601 North American and European isolates of the phylogenetic species PS4 of N. discreta. We found

602 evidence for cryptic population subdivision, underlying a history of strict isolation followed by

603 introgression through secondary contact, with genomic heterogeneity in rates of post-divergence gene

604 flow. Despite the inferred history of long-term isolation, experimental measures of mating success

605 revealed a lack of early intrinsic reproductive barriers between lineages (e.g., mate choice or zygotic

606 mortality), suggesting that the barriers that limit gene flow following secondary contact are mostly

607 extrinsic barriers (e.g., immigrant inviability) or late intrinsic barriers (e.g., hybrid inviability or sterility).

608 In terms of Neurospora biogeography, our study revealed histories of southward migration in North-

609 American lineages, which reinforces previous findings that established Neurospora species as common

610 inhabitants of northern forests, and not only primarily tropical or subtropical fungi as was long believed

611 (Jacobson et al. 2004; Dettman et al. 2006; Perkins \& Turner 1988; Turner et al. 2001a). Further

612 sampling in the northern latitudes should increase our understanding of the diversity and distribution of

613 temperate Neurospora spp. High resolution data sets focusing on currently admixing lineages should

614 also help determining the extent and cause of introgression and in particular whether it is driven by

615 natural selection, either directional or stabilizing. Similar investigations on other models will allow to

616 quantify how common are the factors uncovered in our study in the diversification of fungal lineages.

\section{DATA ACCESSIBILITY}

618 The raw reads were deposited in the Sequence Read Archive and under accession numbers SRS1087846

619 to SRS1087920. Polymorphism data (VCF files), all input data as well as resulting tree files underlying NJ

620 and maximum likelihood phylogenies, mating types and crossing results were deposited in Dryad

621 (doi:10.5061/dryad.1538f). 


\section{ACKNOWLEDGMENTS}

624 We thank Chris Ellison and Sara Branco for assistance with computational analysis, Philipp Messer for

625 sharing his program to perform selective sweep analysis, the Fungal Genetic Stock Center for providing

626 some of the strains used in this study, and Pr. N. Louise Glass for giving access to her lab for Illumina

627 library construction. This work was supported by National Science Foundation grant DEB 1257528 (to

628 JT), a Marie Curie postdoctoral fellowship FP7-PEOPLE-2010-IOF-No.273086 (to PG). BW is member of

629 Dmitri Petrov's lab and was supported by a National Science Foundation graduate research fellowship.

\section{Author Contributions}

631 PG and JT designed research. PG and JT wrote the paper, with contribution from BW and DJ. All authors

632 read and approved the manuscript. DJ and JT collected samples. PG analyzed data, following preliminary

633 analyses by FP, and with contribution from BW for the selective sweep analysis. CHS and PG designed

634 the pipeline for read processing and SNP calling. PG and DK prepared sequencing libraries. MF and IS

635 determined mating types of all isolates, and carried out DNA extraction with PG. LM, DJ and PG

636 conducted the mating compatibility experiment. 


\section{TABLES}

Table 1. Isolates and genome sequencing information

\begin{tabular}{|c|c|c|c|}
\hline Taxon/Origin & $n$ & Proportion of ref. genome covered (\%) & Alignment depth \\
\hline \multicolumn{4}{|l|}{ N. discreta PS4A } \\
\hline Papua New Guinea & $9(9)$ & 80.1 & 22.2 \\
\hline \multicolumn{4}{|l|}{ N. discreta PS4B } \\
\hline Alaska & $8(8)$ & 95.9 & 42.5 \\
\hline California & $15(8)$ & 92.4 & 49.6 \\
\hline New-Mexico & $8(7)$ & 98.7 & 68.8 \\
\hline Washington & $8(6)$ & 96.7 & 75.5 \\
\hline Switzerland & $1(1)$ & 96.9 & 95.1 \\
\hline Spain & $1(1)$ & 96.7 & 38.9 \\
\hline Thailand & $1(1)$ & 86.8 & 25.8 \\
\hline \multicolumn{4}{|l|}{ N. discreta PS6 } \\
\hline Ivory Coast & $1(1)$ & 58.2 & 17.5 \\
\hline
\end{tabular}

$n$ is sample size and between parentheses is the number of genomic sequences included in the study after clone-correction (i.e., after keeping only one representative of nearly identical genomic sequences). 
Table 2. Fixed differences, shared and exclusive polymorphisms.

\begin{tabular}{|l|c|c|c|c|}
\hline Category & $\begin{array}{c}\text { AK-EU vs } \\
\text { CA-WA }\end{array}$ & $\begin{array}{c}\text { AK-EU vs } \\
\text { NM-WA }\end{array}$ & $\begin{array}{c}\text { CA-WA vs } \\
\text { NM-WA }\end{array}$ & $\begin{array}{c}\text { US-EU vs } \\
\text { PS4A }\end{array}$ \\
\hline Total number of biallelic sites & 366958 & 272408 & 354410 & 1172183 \\
\hline Fixed differences & $34.9 \%$ & $43.7 \%$ & $38.6 \%$ & $30.3 \%$ \\
\hline Shared polymorphisms & $5.0 \%$ & $2.4 \%$ & $3.0 \%$ & $1.3 \%$ \\
\hline $\begin{array}{l}\text { Exclusive polymorphisms } \\
\text { lineage 1 }\end{array}$ & $20.3 \%$ & $32.2 \%$ & $43.0 \%$ & $31.2 \%$ \\
\hline $\begin{array}{l}\text { Exclusive polymorphisms } \\
\text { lineage 2 }\end{array}$ & $39.2 \%$ & $21.6 \%$ & $15.0 \%$ & $28.2 \%$ \\
\hline
\end{tabular}

US-EU is the pooling of all three North American/European lineages (i.e., AK-EU $U$ CA-WA $\cup$ NM-WA) 
Table 3. Mean allelic richness (AR) and Mean private allele richness (PAR) per 10kb-window after correction for sample size by rarefaction using ADZE.

\begin{tabular}{|l|l|l|l|}
\hline Lineage & Subpopulation & mean AR (standard error) & mean PAR (standard error) \\
\hline CA-WA & & & \\
\hline & CA & $1.2237(0.0182)$ & $2.2151(0.0087)$ \\
\hline & WA & $1.6714(0.0168)$ & $2.6628(0.0084)$ \\
\hline NM-WA & & & \\
\hline & NM & $2.0907(0.0079)$ & $0.6413(0.0132)$ \\
\hline & WA & $2.4233(0.0089)$ & $0.974(0.0125)$ \\
\hline
\end{tabular}

Sample size was standardized to $n=3$ (size of WA subpopulations). 


\begin{tabular}{|c|c|c|c|c|c|c|c|c|}
\hline Population ID & $n$ & $\pi(\%)$ & $\vartheta w(\%)$ & Tajima's D & He & $\kappa$ & $\rho(\mathrm{M} / \mathrm{kb})$ & LD $50 \%$ decay \\
\hline AK-EU & 10 & $0.098(0.151)$ & $0.093(0.128)$ & $-0.213(0.946)$ & $0.773(0.203)$ & $5.788(2.083)$ & 47.1 & $3 \mathrm{~kb}$ \\
\hline CA-WA & 11 & $0.208(0.216)$ & $0.201(0.193)$ & $-0.035(1.131)$ & $0.88(0.161)$ & 7.91 (2.249) & 24 & $<1 \mathrm{~kb}$ \\
\hline NM-WA & 10 & 0.07 (0.106) & $0.073(0.1)$ & $-0.31(0.873)$ & $0.823(0.166)$ & 6.094 (1.92) & 15.1 & $2 \mathrm{~kb}$ \\
\hline PNG & 9 & $0.318(0.162)$ & $0.36(0.154)$ & $-0.621(0.957)$ & $0.923(0.227)$ & 7.993 (1.955) & 56.4 & $<1 \mathrm{~kb}$ \\
\hline
\end{tabular}

$n$ is sample size; $\vartheta w$ is Watterson's $\vartheta ; \pi$ is nucleotide diversity; He is haplotype diversity; $K$ is the number of haplotypes; $\rho$ is the recombination rate, averaged across the 20 largest scaffolds; $\vartheta w, \pi, H e, K$ are average values across $10 \mathrm{~kb}$ windows with standard deviation between parentheses; LD 50\% decay is the average physical distance over which the coefficient of linkage disequilibrium ( $r 2$ ) decays to half its maximum value. 
Table 5. Likelihood and parameter estimates of an isolation-with-migration model fitted to the data using the python package DADI

\begin{tabular}{|c|c|c|c|}
\hline Dataset/Parameter & Point estimate & Average & SD \\
\hline \multicolumn{4}{|l|}{ PS4A and PS4B-US-EU } \\
\hline Log-likelihood & $-8.13 E+04$ & $-7.96 \mathrm{E}+04$ & $1.13 \mathrm{E}+04$ \\
\hline$N_{\mathrm{AB} 1}$ & $1.41 \mathrm{E}+06$ & $1.39 \mathrm{E}+06$ & $1.98 \mathrm{E}+05$ \\
\hline$m_{\mathrm{PS} 4 \mathrm{~A} \leftarrow \mathrm{PS} 4 \mathrm{BUSEU}}$ & $6.40 \mathrm{E}-08$ & $3.12 \mathrm{E}-06$ & $2.14 \mathrm{E}-05$ \\
\hline$m_{\text {PS4BUSEU }}<$ PS4A & $2.11 \mathrm{E}-42$ & $1.43 \mathrm{E}-12$ & 8.17E-12 \\
\hline$N_{\mathrm{PS} 4 \mathrm{~A} 1}$ & $2.12 \mathrm{E}+05$ & $4.16 \mathrm{E}+05$ & $5.95 E+04$ \\
\hline$N_{\mathrm{PS} 4 \mathrm{~A} 2}$ & $1.58 \mathrm{E}+08$ & $3.07 E+08$ & $4.42 \mathrm{E}+07$ \\
\hline$N_{\text {PS4BUSEU1 }}$ & $8.34 \mathrm{E}+09$ & $2.30 \mathrm{E}+10$ & $1.15 \mathrm{E}+10$ \\
\hline$N_{\text {PS4BUSEU2 }}$ & $9.20 \mathrm{E}+04$ & $1.80 \mathrm{E}+05$ & $2.57 E+04$ \\
\hline$T_{\mathrm{AB} 1}$ & $1.50 \mathrm{E}+06$ & $1.47 \mathrm{E}+06$ & $2.11 \mathrm{E}+05$ \\
\hline$T_{\mathrm{AB} 2}$ & $1.48 \mathrm{E}+06$ & $1.45 \mathrm{E}+06$ & $2.07 E+05$ \\
\hline$T_{\mathrm{AB} 3}$ & $3.61 \mathrm{E}+00$ & $1.70 \mathrm{E}+00$ & $2.12 \mathrm{E}+00$ \\
\hline$T_{\mathrm{AB} 4}$ & $2.94 \mathrm{E}+05$ & $2.87 \mathrm{E}+05$ & $4.10 E+04$ \\
\hline \multicolumn{4}{|c|}{$A K-E U, C A-W A$ and NM-WA lineages within PS4B-US-EU } \\
\hline Log-likelihood & $-7.66 \mathrm{E}+03$ & $-7.77 E+03$ & $1.04 \mathrm{E}+02$ \\
\hline$N_{\mathrm{B}}$ & $1.50 \mathrm{E}+06$ & $1.47 \mathrm{E}+06$ & $3.52 E+04$ \\
\hline$m_{\text {AKEU }} \leftarrow$ CAWA & $3.38 \mathrm{E}-07$ & $3.43 \mathrm{E}-07$ & $1.51 \mathrm{E}-08$ \\
\hline$m_{\text {AKEU }<\text { NMWA }}$ & $3.23 \mathrm{E}-06$ & $3.18 \mathrm{E}-06$ & $8.32 \mathrm{E}-08$ \\
\hline$m_{\text {CAWA }} \leftarrow$ AKEU & 4.55E-08 & 4.15E-08 & 8.23E-09 \\
\hline$m_{\text {CAWA }} \leftarrow$ NMWA & 2.79E-05 & 2.79E-05 & $1.28 \mathrm{E}-06$ \\
\hline$m_{\text {NMWA } \leftarrow \text { AKEU }}$ & $9.30 \mathrm{E}-08$ & $1.02 \mathrm{E}-07$ & $1.05 \mathrm{E}-08$ \\
\hline$m_{\mathrm{NMWA}} \leftarrow$ CAWA & $1.04 \mathrm{E}-08$ & $1.07 \mathrm{E}-08$ & $4.88 \mathrm{E}-09$ \\
\hline$m_{\text {AKEU }<\text { CAWA' }}$ & $1.15 \mathrm{E}-07$ & $1.12 \mathrm{E}-07$ & 4.55E-09 \\
\hline$m_{\text {AKEU } \leftarrow \text { NMWA }}{ }^{\prime}$ & $2.21 \mathrm{E}-07$ & $2.25 \mathrm{E}-07$ & $6.29 \mathrm{E}-09$ \\
\hline$m_{\text {CAWA }} \leftarrow$ AKEU' & $1.87 \mathrm{E}-07$ & $1.86 \mathrm{E}-07$ & 5.71E-09 \\
\hline 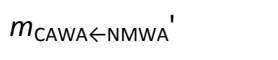 & $2.41 \mathrm{E}-07$ & $2.42 \mathrm{E}-07$ & 7.35E-09 \\
\hline$m_{\text {NMWA } \leftarrow \text { AKEU' }}$ & $3.88 \mathrm{E}-13$ & $6.51 \mathrm{E}-12$ & $3.98 \mathrm{E}-11$ \\
\hline$m_{\text {NMWA }} \leftarrow$ CAWA' & $1.07 \mathrm{E}-07$ & $1.06 \mathrm{E}-07$ & $3.35 \mathrm{E}-09$ \\
\hline$N_{\text {AKEU }}$ & $5.40 \mathrm{E}+04$ & $1.08 \mathrm{E}+05$ & $1.04 \mathrm{E}+03$ \\
\hline$N_{\mathrm{B} 1}$ & $9.14 \mathrm{E}+03$ & $1.75 E+04$ & $1.73 \mathrm{E}+03$ \\
\hline$N_{\text {CAWA }}$ & $1.27 \mathrm{E}+05$ & $2.53 E+05$ & $1.51 \mathrm{E}+03$ \\
\hline$N_{\mathrm{B} 2}$ & $8.73 E-02$ & $1.66 \mathrm{E}-01$ & $1.70 \mathrm{E}-02$ \\
\hline
\end{tabular}




\begin{tabular}{|l|r|r|r|}
\hline$N_{\text {NMWA }}$ & $7.12 \mathrm{E}+04$ & $1.43 \mathrm{E}+05$ & $8.21 \mathrm{E}+02$ \\
\hline$T_{\mathrm{B} 1}$ & $1.30 \mathrm{E}+04$ & $1.21 \mathrm{E}+04$ & $1.39 \mathrm{E}+03$ \\
\hline$T_{\mathrm{B} 2}$ & $8.05 \mathrm{E}-02$ & $7.67 \mathrm{E}-02$ & $7.74 \mathrm{E}-03$ \\
\hline$T_{\mathrm{B} 3}$ & $3.74 \mathrm{E}+05$ & $3.74 \mathrm{E}+05$ & $2.45 \mathrm{E}+03$ \\
\hline$T_{\mathrm{B} 4}$ & $7.40 \mathrm{E}+04$ & $7.42 \mathrm{E}+04$ & $3.35 \mathrm{E}+03$ \\
\hline$p$ & $1.49 \mathrm{E}-01$ & $1.49 \mathrm{E}-01$ & $2.66 \mathrm{E}-03$ \\
\hline
\end{tabular}

Parameter are presented in Figure 3 
Table 6. Summary of genomic divergence between lineages and subpopulation

\begin{tabular}{|c|c|c|c|c|c|}
\hline Pair & $\begin{array}{l}\text { average } F_{S T} \pm \\
\text { S.D. }\end{array}$ & $\begin{array}{l}\text { coefficient of variation } \\
\qquad\left(F_{S T}\right)\end{array}$ & average $d x y \pm$ S.D. & $\begin{array}{l}\text { coefficient of variation } \\
\qquad(d x y)\end{array}$ & Pearson's r ( $P$-value) \\
\hline PS4B-US-EU vs PS4A & $0.650 \pm 0.096$ & 0.147 & $0.01486 \pm 0.00311$ & 0.209 & $0.344(<1 e-92)$ \\
\hline AK-EU vs CA-WA & $0.513 \pm 0.232$ & 0.453 & $0.00358 \pm 0.00341$ & 0.951 & $0.371(<1 \mathrm{e}-107)$ \\
\hline AK-EU vs NM-WA & $0.613 \pm 0.193$ & 0.314 & $0.00253 \pm 0.00274$ & 1.083 & $0.217(<1 e-35)$ \\
\hline CA-WA vs NM-WA & $0.584 \pm 0.206$ & 0.353 & $0.00470 \pm 0.00374$ & 0.796 & $0.227(<1 \mathrm{e}-39)$ \\
\hline$C A$ vs $W A_{(C A-W A)}$ & $0.081 \pm 0.128$ & 1.592 & $0.00207 \pm 0.00220$ & 1.063 & $-0.018(0.297)$ \\
\hline$N M$ vs $W A_{(N M-W A)}$ & $0.089 \pm 0.120$ & 1.349 & $0.00069 \pm 0.00102$ & 1.488 & $-0.047(0.007)$ \\
\hline AK vs EU & $0.116 \pm 0.159$ & 1.366 & $0.00049 \pm 0.00089$ & 1.806 & $-0.006(0.728)$ \\
\hline CA vs NM & $0.613 \pm 0.206$ & 0.336 & $0.00472 \pm 0.00382$ & 0.004 & $0.217(<1 e-35)$ \\
\hline$W A_{(C A-W A)} \operatorname{vs} W A_{(N M-W A)}$ & $0.603 \pm 0.240$ & 0.399 & $0.00547 \pm 0.00435$ & 0.004 & $0.178(<1 \mathrm{e}-24)$ \\
\hline
\end{tabular}


S.D. is the standard deviation. The coefficient of variation is a normalized measure of dispersion calculated as the S.D. to the mean. Pearson'r was used to measure and test the correlation between $F_{S T}$ and relative node depth (Nachman and Payseur 2002). Relative node depth is a measure of divergence that corrects for differences in mutation rate among loci, it is calculated as $d x y$ divided by divergence to an outgroup (here THA). 


\section{FIGURES}

\section{(A)}

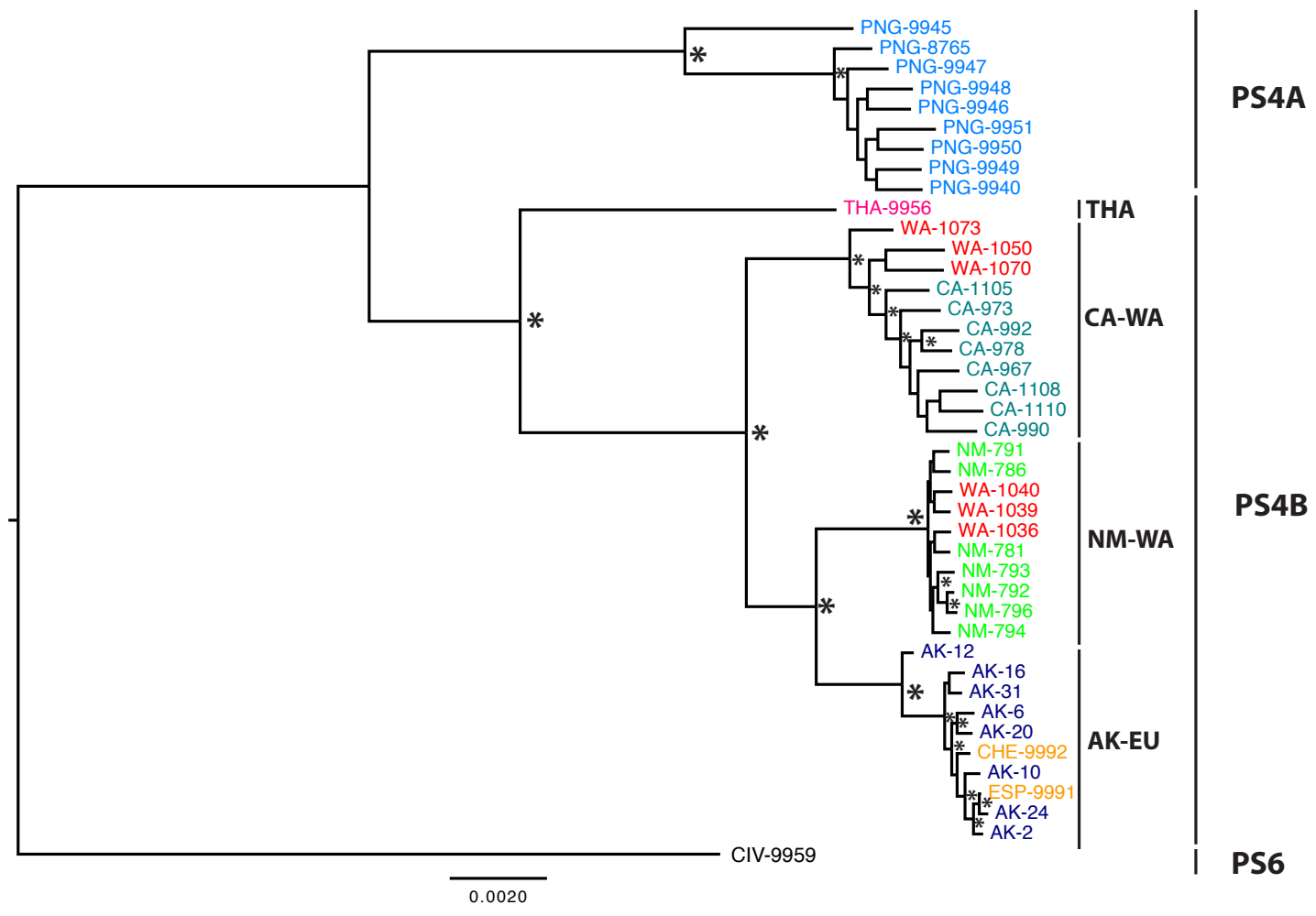

(B)

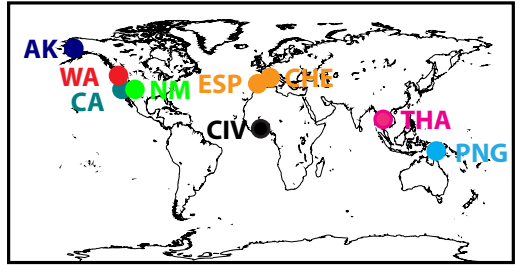

(C)

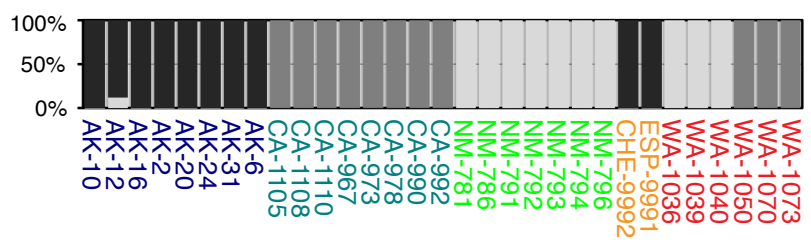

Figure 1. Rooted maximum likelihood genealogy inferred from full genomic sequences (A), Map of sampling sites (B), Cluster-based analysis using FRAPPE (C). In panel A, asterisks denote nodes that received $>99 \%$ bootstrap support, and thin vertical lines denote the different fungal lineages identified. In panel $\mathrm{C}$, each individual is represented by a thick vertical line divided into $\mathrm{K}$ colored segments that represent the individual's estimated membership proportions in the $\mathrm{K}$ clusters assumed (vertical scale at the lef-hand side of the bar plot). In panels $A$ and $C$, individuals IDs are colored according to collection locality (panel B), and individuals ID include ISO country codes and ANSI codes for US states: AK: Alaska, CA: California, WA: Washington, ESP: Spain, CIV: Côte d'Ivoire, CHE: Switzerland, THA: Thailand, PNG: Papua New Guinea. 
(A) AK-EU

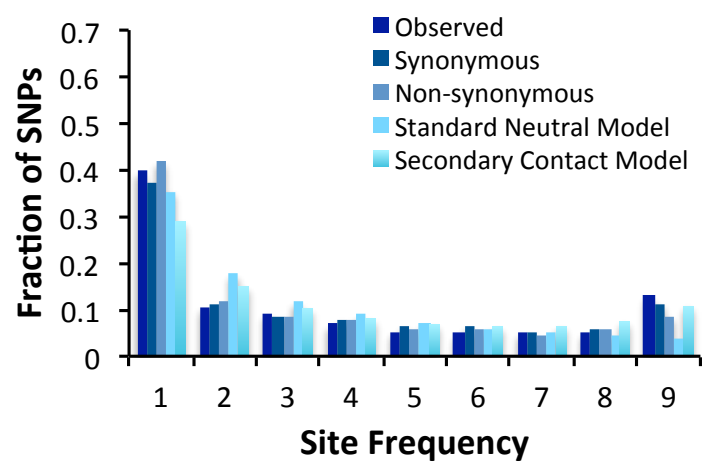

(C) NM-WA

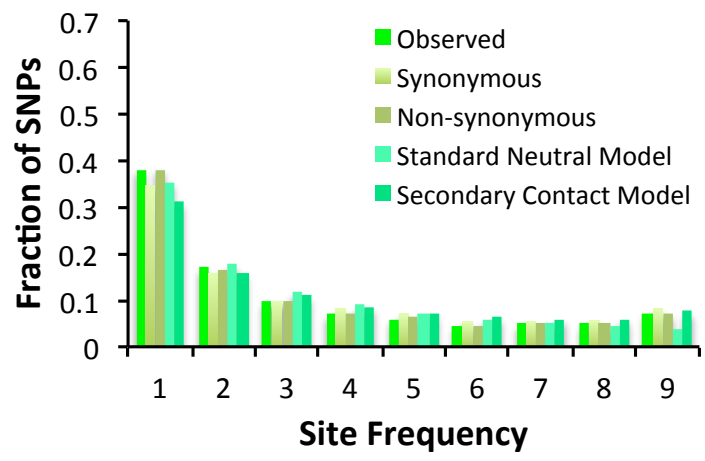

(B) CA-WA

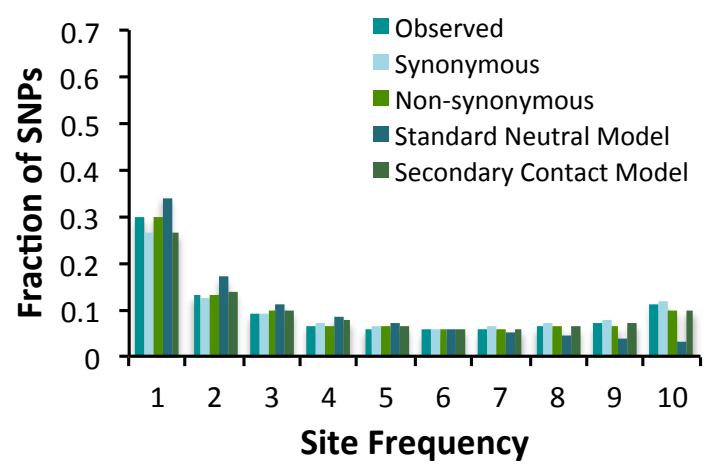

(D) PS4A

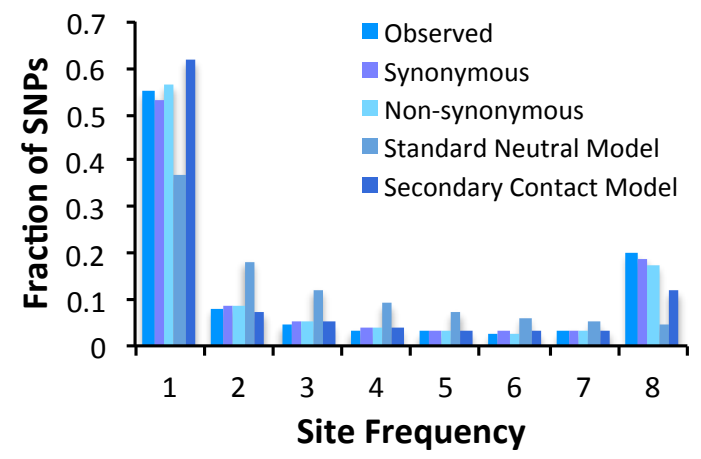

Figure 2. Observed site-frequency-spectra at all sites, synonymous sites or non-synonymous sites, and site-frequency-spectrum fitted using the python package DADI under the standard neutral model or the most supported isolation-with-migration models. The higher fraction of SNPs observed in the data ('Observed') compared to neutral expectations ('Standard Neutral Model') on the left-hand side of the graphs indicates an excess of low frequency derived variants. The lower fraction of SNPs observed in the data ('Observed') compared to neutral expectations ('Standard Neutral Model') on the right-hand side of the graphs indicates an excess of high frequency derived variants. 
(A)

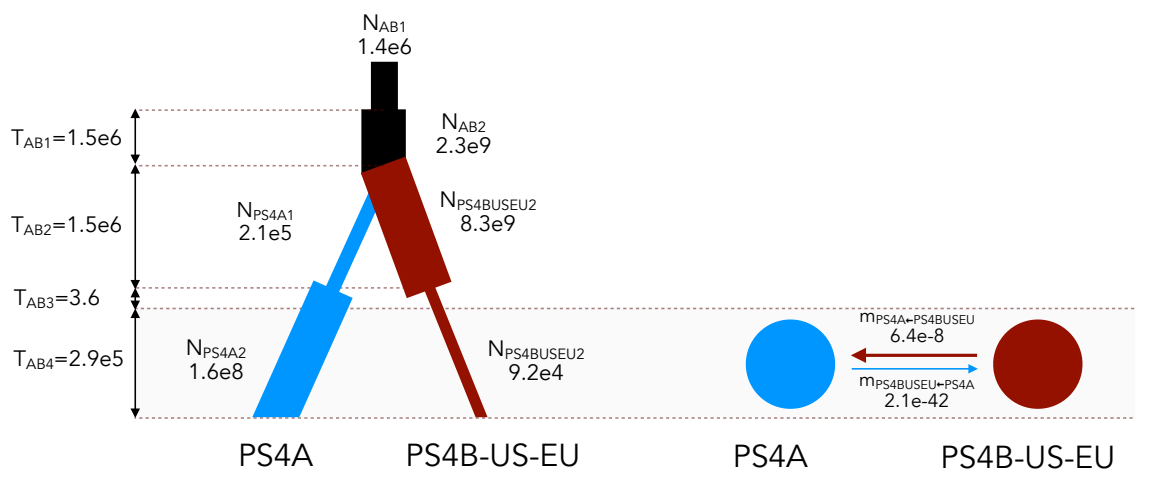

(B)

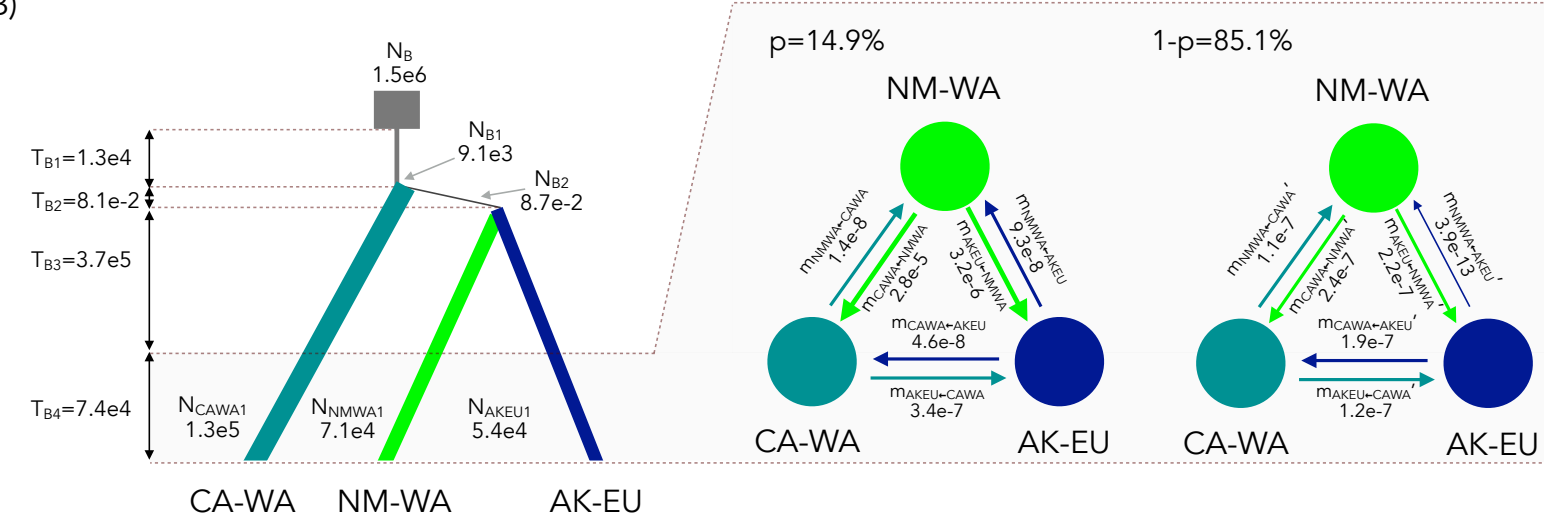

Figure 3. Demographic models of divergence with gene flow (A) between PS4B isolates from the US and Europe (PS4B-US-EU lineages) and lineage PS4A, (B) between lineages within PS4B-US-EU. Population sizes (line width), migration rates (arrow width) and divergence times ( $y$-axis) are not to scale. Times are in number of generations. Migration rates $\left(m_{1<J}\right)$ correspond to the per generation numbers of genetic replacements in pop I by genetic fragments from pop J. For instance, panel (A) presents a demographic model in which the ancestral population (black) experienced an increase in population size before splitting into two lineages (light blue and dark red). One of these latter two lineages experienced an increase in population size (light blue), while the other experienced a decrease (dark red). The two derived lineages were connected by gene flow during $\mathrm{T}_{\mathrm{AB} 4}$ generations, following a period of strict isolation of $\mathrm{T}_{\mathrm{AB} 3}$ generations. Disks and arrows on the right-hand side represent gene flow: for instance, rates of migrations have been much higher from PS4B-US-EU into PS4A (light blue arrow) than the reverse (dark red arrow). 
(A) PS4BUSEU - PS4A

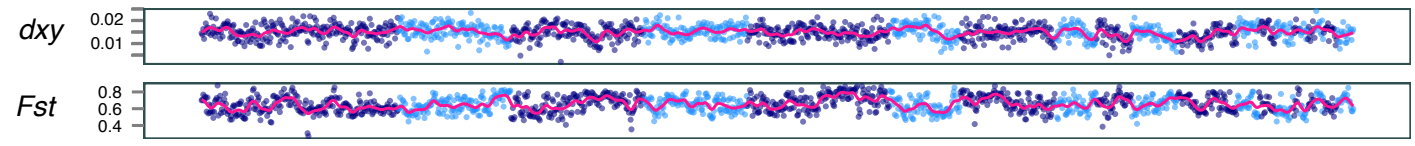

(B) AKEU - CAWA

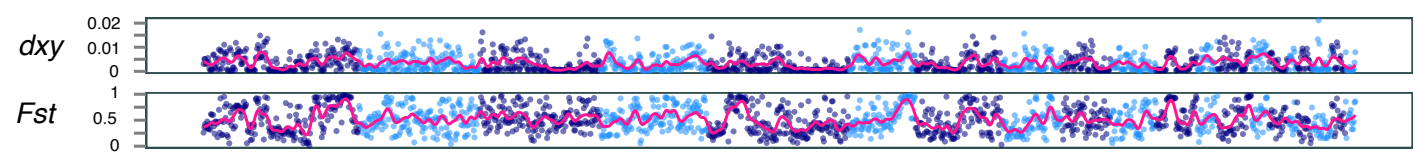

(C) CAWA - NMWA

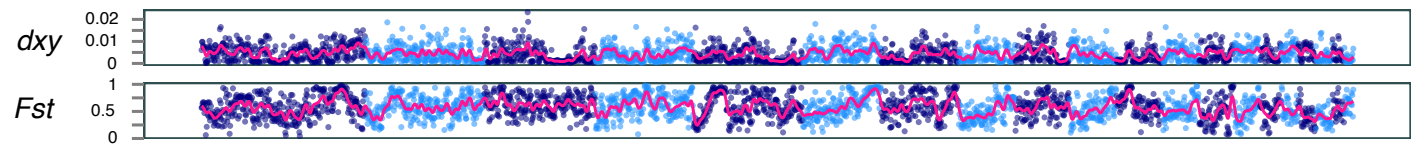

(D) AKEU - NMWA

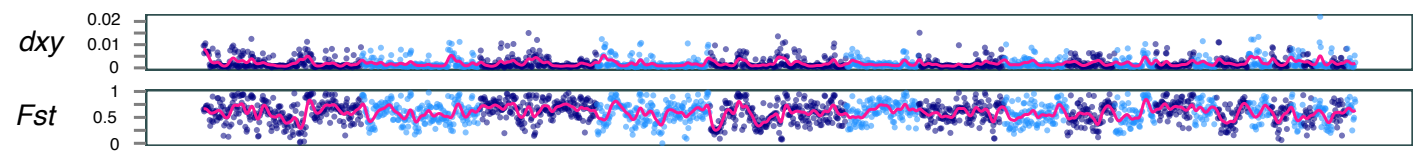

(E) CA - WA

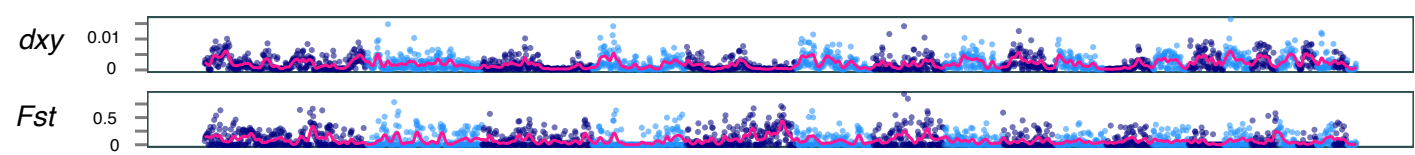

(F) NM - WA

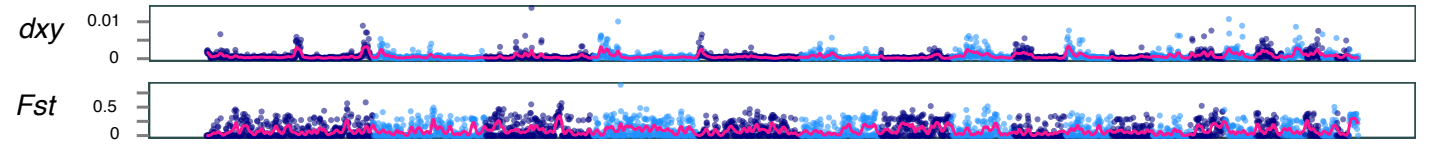

(G) AK - EU

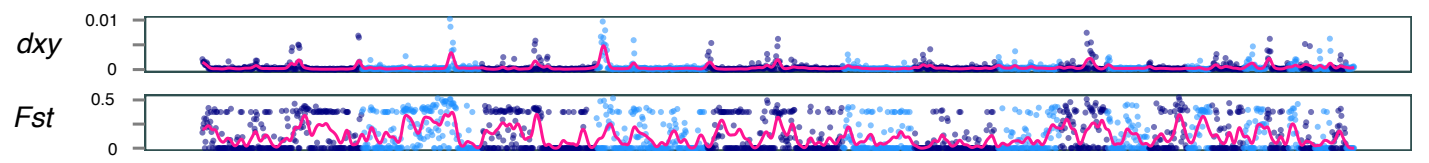

10kb Genomic Windows

Figure 4. Genome-wide distribution of divergence at 10kb windows between pairs of lineages or pairs of subpopulations within lineages. Divergence was estimated in terms of absolute divergence $(d x y)$ and relative divergence (Fst). Only the 20 largest scaffolds are shown. Smooth fitted lines were obtained using a Gaussian kernel method with a bandwidth of 10 . 
(A) Lineages $\rightarrow$

AK-EU

CA-WA

NM-WA Tester

\begin{tabular}{|c|c|c|c|c|c|c|c|c|c|c|c|c|c|}
\hline$\downarrow$ & $a \downarrow A \rightarrow$ & AKFA2 & AKFA24 & AKFA31 & AKFA6 & ES9991 & CH9992 & WACL1050 & WACL1070 & WACL1073 & CAMH1105 & CAWE973 & CAWE978 \\
\hline \multirow{3}{*}{ AK-EU } & AKFA10 & $1 / 1$ & $5 / 5$ & $6 / 6$ & $6 / 6$ & $6 / 5$ & $6 / 6$ & $6 / 6$ & $6 / 6$ & $6 / 6$ & $6 / 6$ & $6 / 6$ & $6 / 6$ \\
\hline & AKFA12 & $6 / 1$ & $5 / 0$ & $6 / 0$ & $6 / 0$ & $5 / 0$ & $6 / 0$ & $6 / 0$ & $6 / 0$ & $6 / 6$ & $6 / 1$ & $6 / 6$ & $6 / 0$ \\
\hline & AKFA20 & $0 / 5$ & $0 / 6$ & $0 / 6$ & $1 / 5$ & $0 / 6$ & $0 / 6$ & $1 / 6$ & $0 / 6$ & $0 / 6$ & $0 / 6$ & $6 / 6$ & $6 / 0$ \\
\hline \multirow{5}{*}{ CA-WA } & CAMH 1108 & $5 / 6$ & $6 / 6$ & $6 / 6$ & $6 / 6$ & $6 / 6$ & $6 / 6$ & $6 / 6$ & $6 / 6$ & $6 / 2$ & $6 / 6$ & $1 / 1$ & $6 / 6$ \\
\hline & CAMH 1110 & $6 / 6$ & $6 / 6$ & $6 / 6$ & $6 / 6$ & $5 / 5$ & $6 / 6$ & $6 / 6$ & $6 / 6$ & $6 / 6$ & $6 / 6$ & $6 / 6$ & $6 / 6$ \\
\hline & CAWE990 & $5 / 5$ & $6 / 6$ & $6 / 6$ & $6 / 6$ & $6 / 5$ & $6 / 6$ & $6 / 6$ & $6 / 6$ & $6 / 6$ & $6 / 6$ & $6 / 6$ & $6 / 6$ \\
\hline & CAWE992 & $5 / 6$ & $5 / 6$ & $6 / 6$ & $6 / 6$ & $6 / 6$ & $5 / 6$ & $6 / 4$ & $6 / 6$ & $6 / 4$ & $6 / 6$ & $6 / 6$ & $6 / 6$ \\
\hline & WACL1039 & $6 / 5$ & $6 / 5$ & $6 / 6$ & $6 / 6$ & $6 / 6$ & $6 / 6$ & $6 / 6$ & $6 / 6$ & $6 / 6$ & $6 / 6$ & $6 / 6$ & $6 / 6$ \\
\hline \multirow{6}{*}{ NM-WA } & NMBE781 & $5 / 0$ & $6 / 6$ & $5 / 0$ & $6 / 1$ & $5 / 0$ & $6 / 0$ & $6 / 0$ & $5 / 5$ & $6 / 0$ & $6 / 0$ & $6 / 0$ & $6 / 0$ \\
\hline & NMBE786 & $6 / 1$ & $6 / 6$ & $6 / 1$ & $6 / 5$ & $6 / 6$ & $6 / 6$ & $6 / 6$ & $6 / 6$ & $6 / 5$ & $6 / 6$ & $6 / 6$ & $6 / 6$ \\
\hline & NMBE792 & $6 / 0$ & $6 / 1$ & $6 / 0$ & $6 / 1$ & $6 / 5$ & $6 / 0$ & $6 / 1$ & $6 / 5$ & $6 / 0$ & $6 / 1$ & $6 / 6$ & $6 / 0$ \\
\hline & NMBE793 & $6 / 2$ & $6 / 5$ & $6 / 5$ & $6 / 0$ & $6 / 2$ & $6 / 6$ & $6 / 1$ & $6 / 1$ & $6 / 4$ & $6 / 6$ & $6 / 6$ & $6 / 6$ \\
\hline & NMBE794 & $5 / 0$ & $6 / 6$ & $6 / 0$ & $6 / 0$ & $2 / 5$ & $6 / 0$ & $6 / 0$ & $6 / 0$ & $6 / 0$ & $6 / 0$ & $6 / 5$ & $6 / 0$ \\
\hline & NMBE796 & $6 / 2$ & $6 / 0$ & $6 / 0$ & $6 / 6$ & $6 / 6$ & $6 / 0$ & $6 / 0$ & $0 / 6$ & $0 / 5$ & $6 / 6$ & $6 / 6$ & $6 / 6$ \\
\hline Tester & TX8127 & $6 / 1$ & $6 / 6$ & $5 / 6$ & $6 / 6$ & $6 / 6$ & $6 / 6$ & $6 / 6$ & $6 / 6$ & $6 / 6$ & $6 / 6$ & $6 / 6$ & $6 / 6$ \\
\hline
\end{tabular}

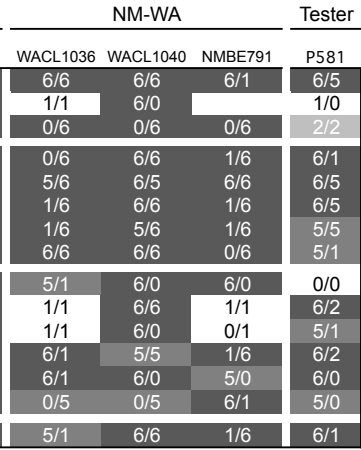

(B)

\begin{tabular}{|c|lcc|}
\hline & & \multicolumn{2}{c|}{ Category of Reproductive Success } \\
\hline 6 & $>50 \%$ black ascospores & 2 & perithecia developed ostioles, no spores \\
5 & $15-50 \%$ black ascospores & $0 \& 1$ & sterile, no perithecia produced; \& barren perithecia, no ostiole developed \\
$3 \& 4$ & $<1 \%$ black ascospores $; \& 1-15 \%$ black ascospores & \\
\hline
\end{tabular}

Figure 5. (A) Reproductive success of 239 crosses (478 matings). Columns represent the 16 mat $A$ strains, and rows represent the 15 mat a strains, with isolates numbers along the row and column headings of the matrix. Numbers within matrix cells indicate the reproductive success ratings (see $B$ ) of the two reciprocal matings of the cross between the corresponding isolates (mat $a$ isolate as the perithecial parent/mat $A$ isolate as the perithecial parent). The matrix cells have been shaded in proportion to the reproductive success of the best mating (see $B$ ). Additional row and column headings indicate the lineage designation (see Figure 1). Isolates P581 and TX8127 are tester strains of $N$. discreta sensu stricto. (B) Categories of reproductive success, corresponding to different stages in reproductive development, from the development of perithecia, to the formation of black ascospores. 


\begin{tabular}{|c|c|c|c|c|c|c|}
\hline Taxon/Isolate & Origin & $\begin{array}{l}\% \text { reference } \\
\text { sequence } \\
\text { covered }\end{array}$ & $\begin{array}{l}\text { sequencing } \\
\text { depth }\end{array}$ & $\begin{array}{l}\text { standard } \\
\text { deviation of } \\
\text { sequencing } \\
\text { depth }\end{array}$ & $\begin{array}{l}\text { included in } \\
\text { clone } \\
\text { corrected } \\
\text { dataset }\end{array}$ & $\begin{array}{l}\text { SRA accession } \\
\text { number }\end{array}$ \\
\hline \multicolumn{7}{|c|}{ N. discreta PS4B } \\
\hline AK-10 & Fairbanks, Alaska & 93.2 & 24.1 & 43 & yes & SRS1087866 \\
\hline AK-12 & Fairbanks, Alaska & 97.5 & 37.8 & 16.9 & yes & SRS1087856 \\
\hline AK-16 & Fairbanks, Alaska & 96.2 & 42.6 & 28.3 & yes & SRS1087865 \\
\hline AK-2 & Fairbanks, Alaska & 96.5 & 61.6 & 27.6 & yes & S SRS1087873 \\
\hline AK-20 & Fairbanks, Alaska & 96.2 & 40.9 & 19.3 & yes & SRS1087864 \\
\hline AK-24 & Fairbanks, Alaska & 95.1 & 25.2 & 55.3 & yes & SRS1087855 \\
\hline AK-31 & Fairbanks, Alaska & 96.3 & 46.2 & 23.2 & yes & SRS1087863 \\
\hline AK-6 & Fairbanks, Alaska & 96.4 & 61.4 & 25.6 & yes & SRS1087854 \\
\hline CA-1099 & Morgan Hill, California & 89.9 & 23.3 & 58.4 & no & o SRS1087872 \\
\hline CA-1100 & Morgan Hill, California & 94 & 40.3 & 20.1 & no & o SRS1087857 \\
\hline CA-1101 & Morgan Hill, California & 94.1 & 39.9 & 22.3 & no & o SRS1087862 \\
\hline CA-1102 & Morgan Hill, California & 94.4 & 49.4 & 23.6 & yes & SRS1087852 \\
\hline CA-1105 & Morgan Hill, California & 94.1 & 50.3 & 21.6 & yes & SRS1087851 \\
\hline CA-1106 & Morgan Hill, California & 94.1 & 43.9 & 23.6 & no & o SRS1087861 \\
\hline CA-1108 & Morgan Hill, California & 94.4 & 35.4 & 20.4 & yes & S SRS1087860 \\
\hline CA-1110 & Morgan Hill, California & 94.7 & 62.5 & 28.2 & yes & SRS1087850 \\
\hline CA-1001 & Weaverville, California & 81.9 & 14.3 & 42.2 & no & o SRS1087867 \\
\hline CA-967 & Weaverville, California & 94.5 & 74.8 & 29.3 & yes & S SRS1087920 \\
\hline CA-973 & Weaverville, California & 96 & 89.1 & 38.9 & yes & S SRS1087910 \\
\hline CA-978 & Weaverville, California & 94.6 & 73.8 & 30.1 & yes & S SRS1087913 \\
\hline CA-990 & Weaverville, California & 94.7 & 108.6 & 41.2 & yes & S SRS1087915 \\
\hline CA-992 & Weaverville, California & 92.5 & 25.6 & 44.9 & yes & S SRS1087847 \\
\hline CA-995 & Weaverville, California & 82.7 & 12.7 & 37.3 & no & o SRS1087858 \\
\hline NM-778 & Belen, New Mexico & 98.7 & 83.7 & 26.6 & no & o SRS1087871 \\
\hline NM-781 & Belen, New Mexico & 98.8 & 81.1 & 26.4 & yes & S SRS1087870 \\
\hline NM-786 & Belen, New Mexico & 98.8 & 71.6 & 24.2 & yes & S SRS1087849 \\
\hline NM-791 & Belen, New Mexico & 98.7 & 54.6 & 16.6 & yes & S SRS1087859 \\
\hline NM-792 & Belen, New Mexico & 98.7 & 52.6 & 19.2 & yes & S SRS1087846 \\
\hline NM-793 & Belen, New Mexico & 98.7 & 57.8 & 18.5 & yes & S SRS1087868 \\
\hline NM-794 & Belen, New Mexico & 98.7 & 62.7 & 17.8 & yes & S SRS1087848 \\
\hline NM-796 & Belen, New Mexico & 98.6 & 86.5 & 27.7 & yes & S SRS1087869 \\
\hline ESP-9991 & Macanet de la Selva, Spain & 96.7 & 104.1 & 38.9 & yes & S SRS1087914 \\
\hline THA-9956 & Khao Yai, Thailand & 86.8 & 25.8 & 16.8 & yes & S SRS1087909 \\
\hline WA-1036 & Lake Chelan, Washington & 98.7 & 90 & 25.7 & yes & S SRS1087916 \\
\hline WA-1039 & Lake Chelan, Washington & 98.8 & 99.5 & 28.6 & yes & SRS1087919 \\
\hline WA-1040 & Lake Chelan, Washington & 98.9 & 76.2 & 43.6 & yes & SRS1087900 \\
\hline WA-1050 & Lake Chelan, Washington & 94.8 & 46.9 & 47.3 & yes & SRS1087899 \\
\hline WA-1056 & Lake Chelan, Washington & 94.3 & 84 & 32.9 & no & o SRS1087917 \\
\hline WA-1068 & Lake Chelan, Washington & 98.8 & 84.3 & 47.2 & no & o SRS1087911 \\
\hline WA-1070 & Lake Chelan, Washington & 94.9 & 78.9 & 49 & yes & SRS1087875 \\
\hline WA-1073 & Lake Chelan, Washington & 94.5 & 44 & 27.8 & yes & SRS1087853 \\
\hline CHE-9992 & Leuk, Switzerland & 96.9 & 95.1 & 32.6 & yes & SRS1087912 \\
\hline
\end{tabular}

N. discreta PS4A 


\begin{tabular}{llrrrr} 
PNG-8765 & Tiaba, Papua New Guinea & 79 & 13.9 & 11.2 & yes SRS1087908 \\
\hline PNG-9940 & Tiaba, Papua New Guinea & 80.5 & 21.7 & 16.5 & yes SRS1087907 \\
\hline PNG-9945 & Wau, Papua New Guinea & 79.9 & 17.5 & 14 & yes SRS1087906 \\
\hline PNG-9946 & Marinville, Papua New Guinea & 78.7 & 15.6 & 12.4 & yes SRS1087905 \\
PNG-9947 & Sogeri Road, Papua New Guinea & 77.8 & 12.4 & 9.9 & yes SRS1087904 \\
\hline PNG-9948 & Sogeri Road, Papua New Guinea & 80.1 & 22 & 16.8 & yes SRS1087903 \\
PNG-9949 & Rouna, Papua New Guinea & 80 & 20.2 & 15.4 & yes SRS1087902 \\
PNG-9950 & Rouna, Papua New Guinea & 80.3 & 19.3 & 14.9 & yes SRS1087901 \\
PNG-9951 & Hiri, Papua New Guinea & 84.3 & 56.9 & 40.4 & yes SRS1087918 \\
\hline N. discreta PS6 & & & & & yes SRS1087874 \\
\hline CIV-9959 & Gahelile, Côte d'Ivoire & 58.2 & 17.5 & 25.2 & \\
\hline
\end{tabular}




\begin{tabular}{|c|c|c|c|}
\hline Dataset/Model & Free parameters & Ln L & AIC \\
\hline \multicolumn{4}{|l|}{ PS4A and PS4B-US-EU* } \\
\hline No migration, constant population sizes & 4 & -225655 & 451318 \\
\hline Migration, constant population sizes & 6 & -165782 & 331576 \\
\hline Migration, size change in ancestral population & 7 & -162765 & 325544 \\
\hline Ancient migration, size change in ancestral population & 8 & -163345 & 326706 \\
\hline Secondary contact, size change in ancestral population & 8 & -153321 & 306658 \\
\hline Secondary contact, heterogeneous migration, size change in ancestral population*** & 11 & -153321 & 306664 \\
\hline Secondary contact, size change in ancestral population and simultaneously in both lineages & 11 & -81314 & 162650 \\
\hline Migration, size change in ancestral population and sequentially in lineages PS4A and PS4B-US-EU**** & 12 & -81314 & 162652 \\
\hline Migration, size change in ancestral population and sequentially in lineages PS4B-US-EU and PS4A**** & 12 & -81314 & 162652 \\
\hline \multicolumn{4}{|l|}{$A K-E U, C A-W A$ and NM-WA lineages within PS4B-US-EU** } \\
\hline No migration, constant population sizes & 6 & -21891 & 43794 \\
\hline Continuous migration, constant population sizes & 12 & -15475 & 30974 \\
\hline Continuous migration, size change in ancestral population & 14 & -12044 & 24116 \\
\hline Continuous migration, size change in ancestral population and along interior branch & 16 & -12045 & 24122 \\
\hline Ancient migration, size change in ancestral population & 15 & -12099 & 24228 \\
\hline Secondary contact, size change in ancestral population & 15 & -11105 & 22240 \\
\hline Secondary contact, heterogeneous migration, size change in ancestral population & 22 & -7658 & 15360 \\
\hline Secondary contact, heterogeneous migration, size change in ancestral population and simultaneously in all three lineages & 26 & -9000 & 18052 \\
\hline Secondary contact, heterogeneous migration, size change in ancestral population and sequentially in lineages AK-EU, CA-WA and NM-WA & 28 & -9000 & 18056 \\
\hline Secondary contact, heterogeneous migration, size change in ancestral population and sequentially in lineages AK-EU, NM-WA and CA-WA & 28 & -9000 & 18056 \\
\hline Secondary contact, heterogeneous migration, size change in ancestral population and sequentially in lineages CA-WA, AK-EU and NM-WA & 28 & -9000 & 18056 \\
\hline Secondary contact, heterogeneous migration, size change in ancestral population and sequentially in lineages CA-WA, NM-WA and AK-EU & 28 & -9000 & 18056 \\
\hline Secondary contact, heterogeneous migration, size change in ancestral population and sequentially in lineages NM-WA, AK-EU and CA-WA & 28 & -9000 & 18056 \\
\hline Secondary contact, heterogeneous migration, size change in ancestral population and sequentially in lineages NM-WA, CA-WA and AK-EU & 28 & -9000 & 18056 \\
\hline
\end{tabular}

Secondary contact, heterogeneous migration, size change in ancestral population and sequentially in lineages NM-WA, CA-WA and AK-EU

*PS4B-US-EU corresponds to the pooling of lineages AK-EU, CA-WA and NM-WA

**All models assumed the topology ((AK-EU,NM-WA),CA-WA) as the genealogical relationship among lineages. The 'interior branch' is the branch holding lineages AK-EU and NM-WA.

$* * *$ identical to model without heterogeneous migration, as the estimate was zero for the $p$ parameter modelling the relative proportion of two classes of migration rates

$* * * *$ identical to model with simultaneous size change, as the estimate was zero for the interval between the two size changes 
Table S3. Genomic coordinates of peaks of highest average pairwise homozygosity $\mathrm{H}$ as measured by the product of average pairwise homozygosity tract length extending in both directions from each SNP and the number of SNPs in each pairwise homozygous region to account for variable SNP density ( $b p \times S)$. The corresponding lengths of the region of high average pairwise homozygosity are

\begin{tabular}{|c|c|c|c|c|c|}
\hline Lineage & Scaffold & & dinate & $H(b p)$ & $H(b p \times s)$ \\
\hline AKEU & & 2 & 2589111 & 6109.27 & $6.81 \mathrm{E}+07$ \\
\hline AKEU & & 7 & 156165 & 12629.2 & 7.37E+07 \\
\hline AKEU & & 8 & 1250434 & 10103.2 & $7.61 E+07$ \\
\hline CAWA & & 3 & 2066258 & 8291.8 & $1.27 E+08$ \\
\hline CAWA & & 7 & 354872 & 11756.2 & $8.16 E+07$ \\
\hline CAWA & & 4 & 1340904 & 19909.4 & $5.85 E+07$ \\
\hline NMWA & & 1 & 838281 & 8826.13 & $1.55 E+09$ \\
\hline NMWA & & 4 & 731500 & 2342.6 & $1.85 E+09$ \\
\hline NMWA & & 7 & 187 & 6384.73 & $1.65 E+09$ \\
\hline PS4A & & 1 & 1409210 & 1300.67 & $5.48 E+06$ \\
\hline PS4A & & 3 & 2180404 & 5729.94 & $4.81 E+06$ \\
\hline PS4A & & 5 & 2528828 & 7681.25 & $3.64 E+06$ \\
\hline PS4B-US-EU & & 1 & 843407 & 2626.09 & $1.58 \mathrm{E}+09$ \\
\hline PS4B-US-EU & & 7 & 231261 & 8213.07 & $6.08 E+08$ \\
\hline PS4B-US-EU & & 2 & 455323 & 2506.37 & $5.80 E+08$ \\
\hline
\end{tabular}




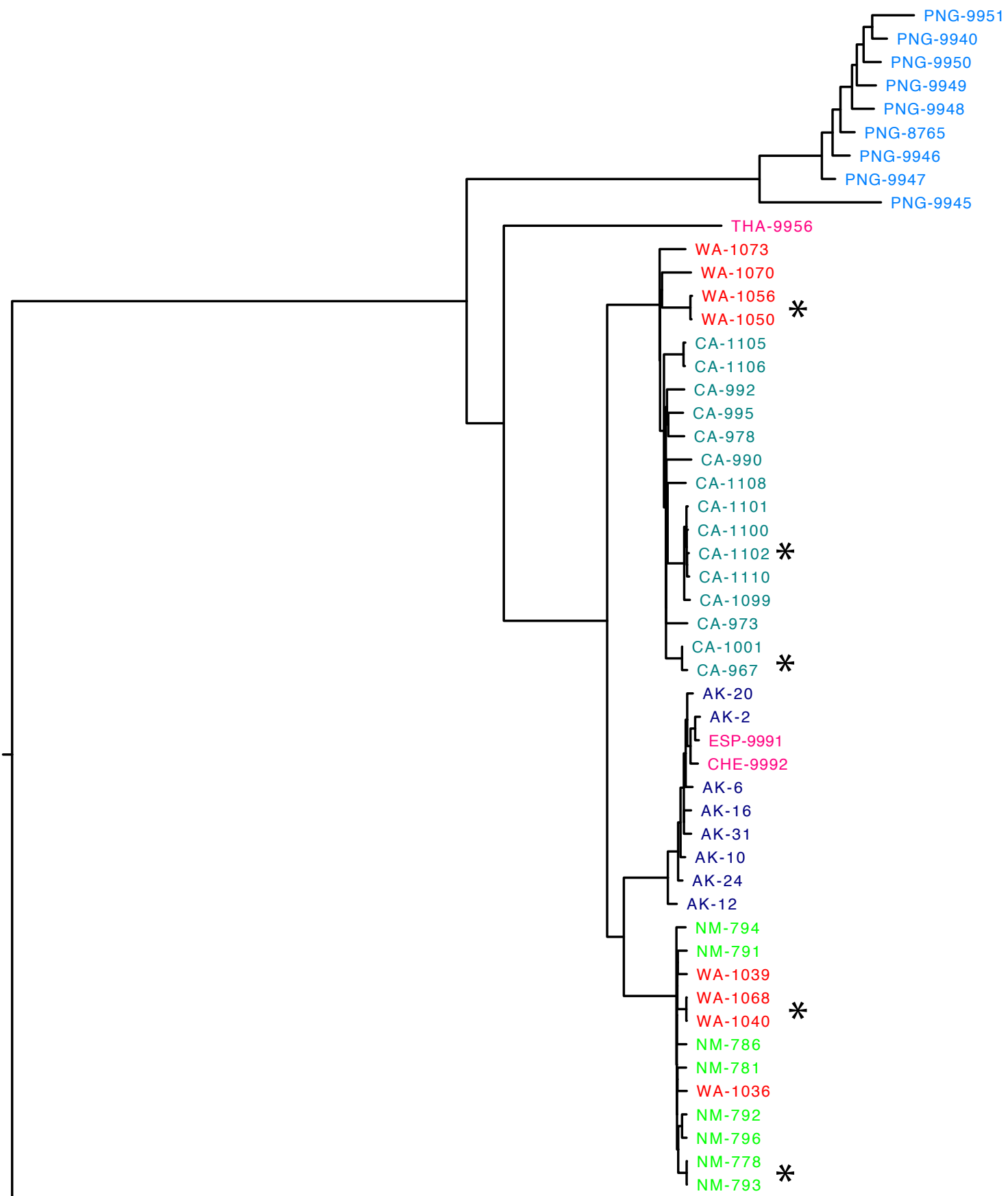

Supplementary Figure S1. Neighbor-joining based on Felsenstein's 1984 genetic distance, calculated on the full set of SNPs. Asterisks indicate nearly identical genotypes that were subjected to clone-correction. 


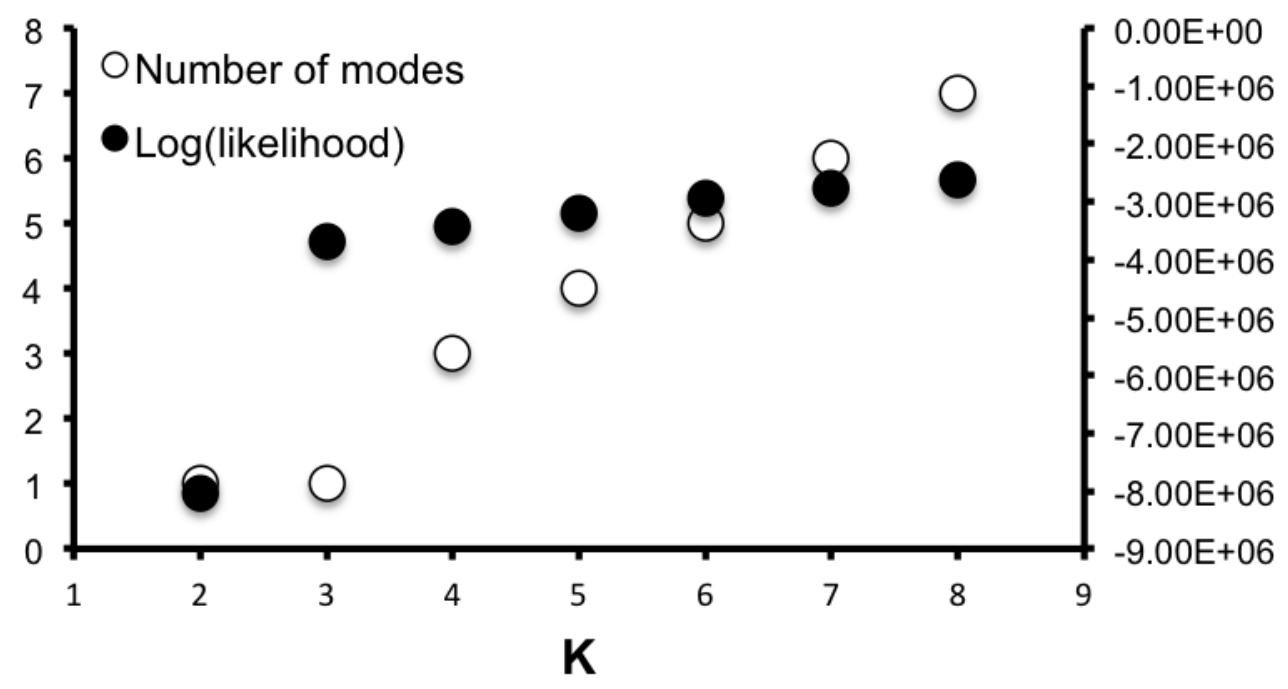

Figure S2. Numbers of modes (i.e., clustering solutions) and average likelihoods over 10 Frappe replicated runs plotted as a function of the putative number of clusters (K). 


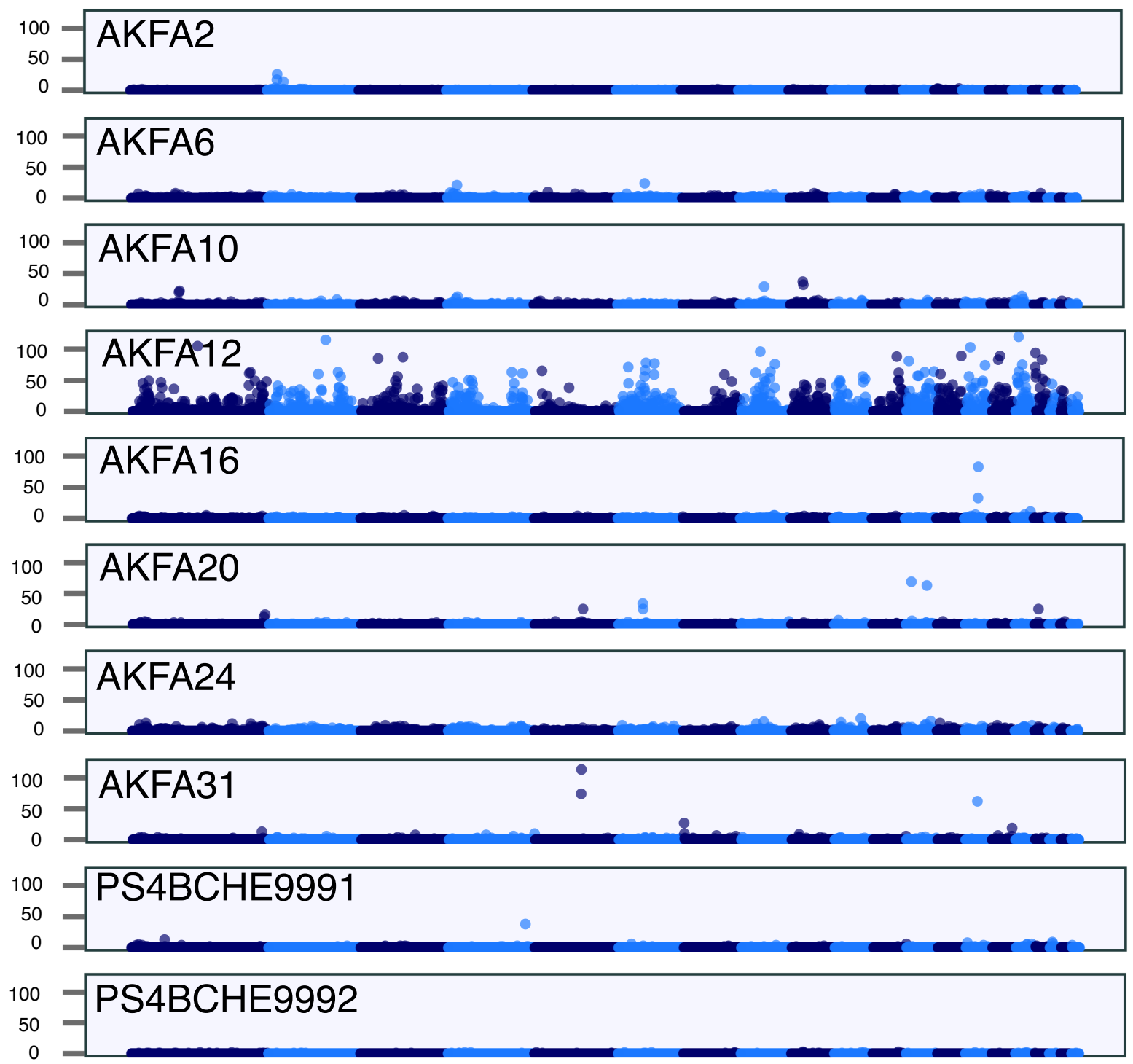

\section{0kb Genomic windows}

Figure S3. Genome-wide distribution of the number of candidate introgressed variants per $10 \mathrm{~kb}$ windows in isolates of the AK-EU lineage. For a focal isolate, candidate introgressed variants are SNPs which are fixed for alternate alleles between the NM-WA isolates and the focal isolate on the one hand, and the rest of AK-EU isolates on the other hand. Only the 20 largest scaffolds are presented. On average, the number of candidate introgressed variants was 4.95 per $10 \mathrm{~kb}$ window for isolate AKFA12 and less than 0.47 for other isolates. 
(B)
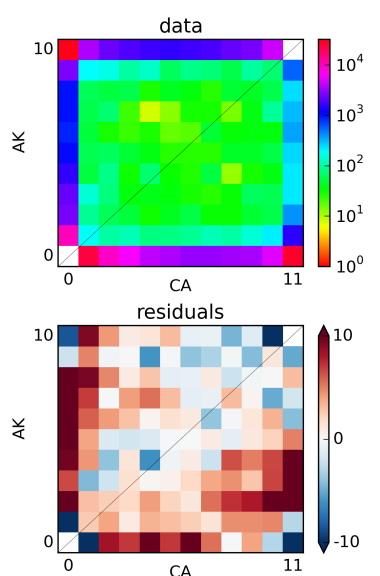

(C)
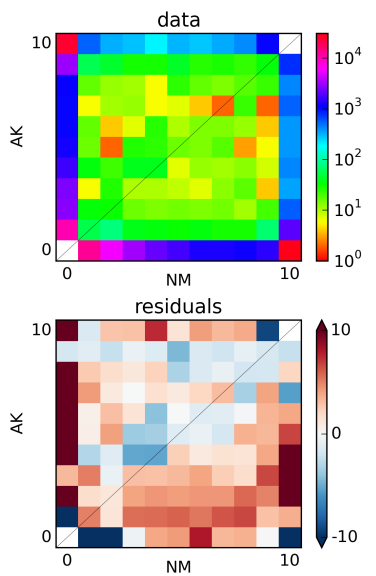
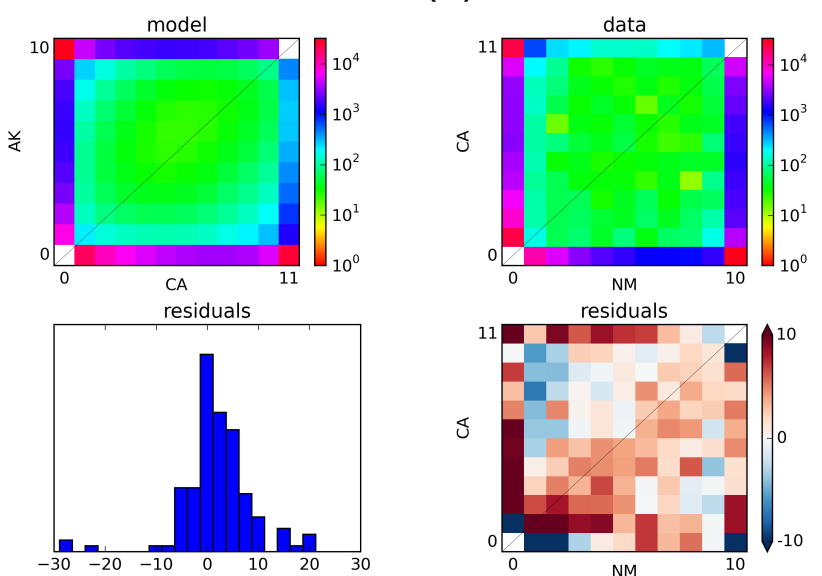

(D)

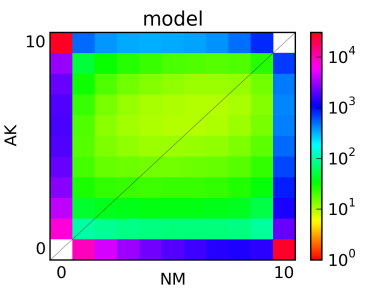

residuals

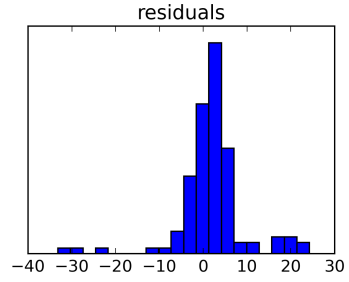

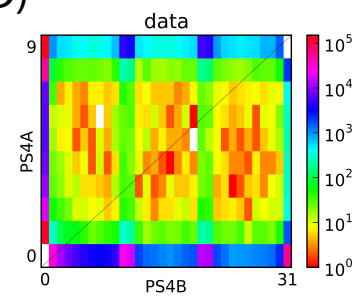

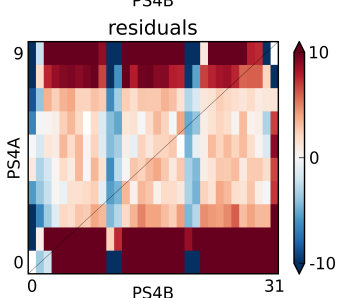

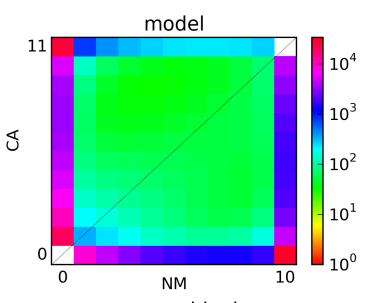

residuals
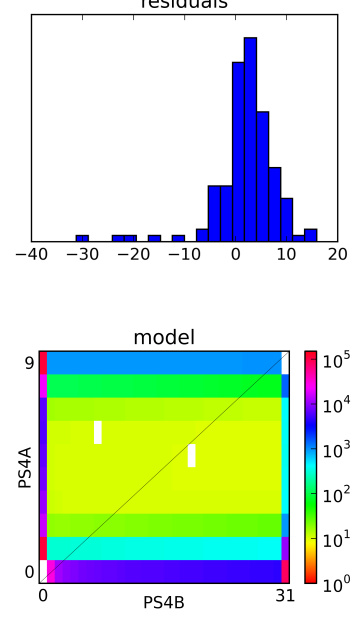

residuals

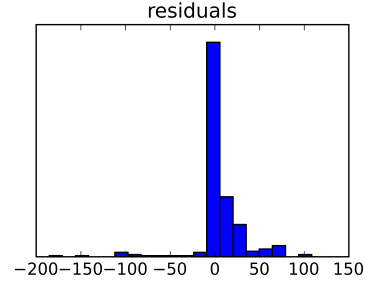

Figure S4. Comparison of the observed joint allele frequency spectrum with that expected under the demographic model fitted using dadi, and corresponding residuals. The 3-dimensional Joint frequency spectrum is represented as three 2-dimensional marginal spectra plotted using heat-maps: (A) AK-EU vs CA-WA, (B) CA-WA vs NM-WA, (C) AK-EU vs NM-WA and (D) PS4B-US-EU vs PS4A, with PS4B-US-EU corresponding to the pooling of AK-EU, CA-WA and NM-WA. The color of a cell at position $(x, y)$ in the marginal spectrum corresponds to the number of derived alleles that are at frequency $x$ in one lineage and frequency $y$ in the other. The residuals represent the difference in the number of alleles predicted by the model compared with that found in the data for each bin in the spectrum (red, model predicts too many; blue, model predicts too few). 
(A) AK-EU

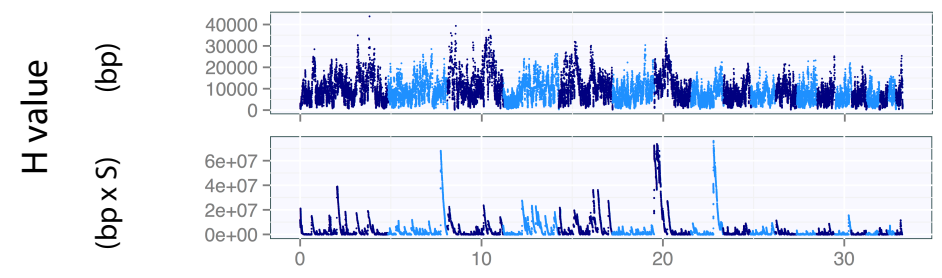

(B) CA-WA

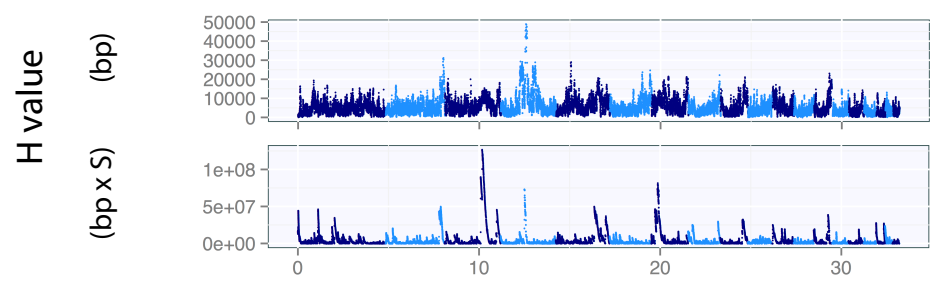

(C) NM-WA

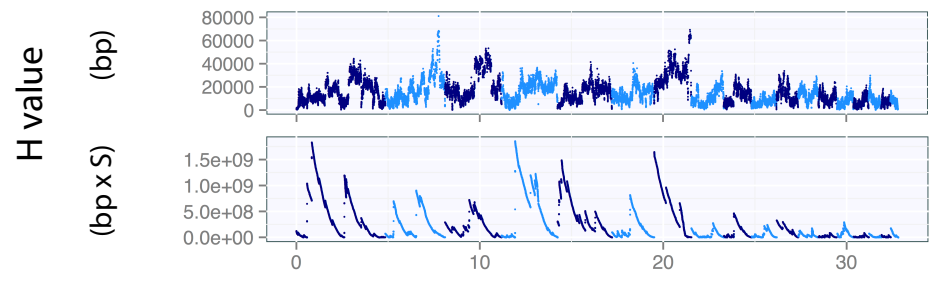

(D) PS4A-PNG

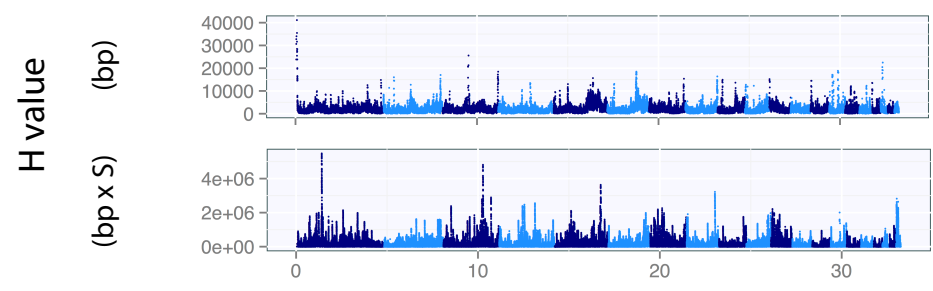

(E) PS4B-US-EU

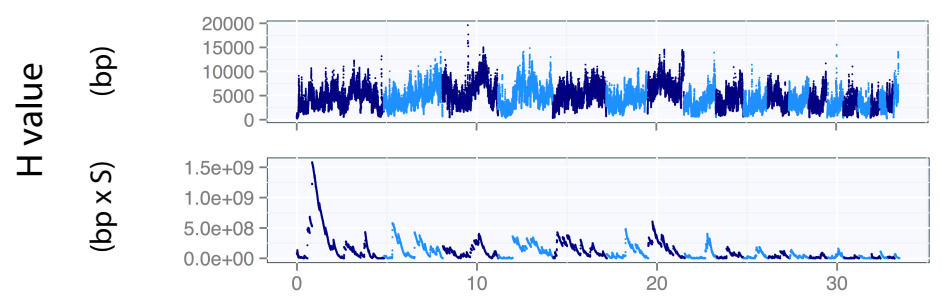

Genomic coordinates (Mbp)

Figure S5. H-scan for recent selective sweeps along scaffolds of four lineages of $N$. discreta PS4. Only scaffolds with more than 100 variable sites were considered (i.e., the 18 to 20 longest scaffolds, representing $88 \%$ to $90 \%$ of the full genome size). Each dot represents the $\mathrm{H}$ statistic value calculated at site that is variable within a given lineage. 


\section{REFERENCES}

Auton A, McVean G (2007) Recombination rate estimation in the presence of hotspots. Genome Research 17, 1219-1227.

Barnett DW, Garrison EK, Quinlan AR, Strömberg MP, Marth GT (2011) BamTools: a C++ API and toolkit for analyzing and managing BAM files. Bioinformatics 27, 1691-1692.

Barton NH, Bengtsson BO (1986) The barrier to genetic exchange between hybridising populations. Heredity 57, 357-376.

Barton NH, Hewitt GM (1981) Hybrid zones and speciation. In: Evolution and Speciation, pp. 109-145. Cambridge University Press, Cambridge.

Brand CL, Kingan SB, Wu L, Garrigan D (2013) A selective sweep across species boundaries in Drosophila. Molecular Biology and Evolution 30, 2177-2186.

Corcoran P, Dettman JR, Sun Y, et al. (2014) A global multilocus analysis of the model fungus Neurospora reveals a single recent origin of a novel genetic system. Molecular Phylogenetics and Evolution 78, 136-147.

Coyne JA, Orr HA (2004) Speciation Sinauer Associates Sunderland, MA.

Danecek P, Auton A, Abecasis G, et al. (2011) The variant call format and VCFtools. Bioinformatics 27, 2156-2158.

Davis RH, Perkins DD (2002) Neurospora: a model of model microbes. Nature Reviews Genetics 3, 397403.

Dettman J, Jacobson D, Taylor J (2003) A multilocus genealogical approach to phylogenetic species recognition in the model eukaryoteNeurospora. Evolution 57, 2703-2720.

Dettman JR, Anderson JB, Kohn LM (2008) Divergent adaptation promotes reproductive isolation among experimental populations of the filamentous fungus Neurospora. Bmc Evolutionary Biology 8 , 35.

Dettman JR, Jacobson DJ, Taylor JW (2006) Multilocus sequence data reveal extensive phylogenetic species diversity within the Neurospora discreta complex. Mycologia 98, 436-446.

Dettman JR, Jacobson DJ, Turner E, Pringle A, Taylor JW (2003) Reproductive isolation and phylogenetic divergence in Neurospora: comparing methods of species recognition in a model eukaryote. Evolution 57, 2721-2741.

Dettman JR, Rodrigue N, Melnyk AH, et al. (2012) Evolutionary insight from whole-genome sequencing of experimentally evolved microbes. Molecular Ecology 21, 2058-2077.

Ellison CE, Hall C, Kowbel D, et al. (2011) Population genomics and local adaptation in wild isolates of a model microbial eukaryote. Proceedings of the National Academy of Sciences 108, 2831-2836. 
Fay JC, Wu C-I (2000) Hitchhiking under positive Darwinian selection. Genetics 155, 1405-1413.

Fontaine MC, Gladieux P, Hood ME, Giraud T (2013) History of the invasion of the anther smut pathogen on Silene latifolia in North America. New Phytologist 198, 946-956.

Garrigan D, Kingan SB, Geneva AJ, et al. (2012) Genome sequencing reveals complex speciation in the Drosophila simulans clade. Genome Research 22, 1499-1511.

Gillespie JH (2001) Is the population size of a species relevant to its evolution? Evolution 55, 2161-2169.

Gladieux P, Guerin F, Giraud T, et al. (2011) Emergence of novel fungal pathogens by ecological speciation: importance of the reduced viability of immigrants. Molecular Ecology 20, 4521-4532.

Glass NL, Grotelueschen J, Metzenberg RL (1990) Neurospora crassa A mating-type region. Proceedings of the National Academy of Sciences 87, 4912-4916.

Gutenkunst RN, Hernandez RD, Williamson SH, Bustamante CD (2009) Inferring the joint demographic history of multiple populations from multidimensional SNP frequency data. Plos Genetics 5, e1000695.

Hill WG, Robertson A (1968) Linkage disequilibrium in finite populations. Theoretical and Applied Genetics 38, 226-231.

Houston DB (1973) Wildfires in Northern Yellowstone National Park. Ecology 54, 1111-1117.

Jacobson DJ (1995) Sexual dysfunction associated with outcrossing in Neurospora tetrasperma, a pseudohomothallic ascomycete. Mycologia, 604-617.

Jacobson DJ, Powell AJ, Dettman JR, et al. (2004) Neurospora in temperate forests of western North America. Mycologia 96, 66-74.

Jobling MA, Hurles ME, Tyler-Smith C (2004) Human Evolutionary Genetics: Origins, Peoples \& Disease Garland Science Publishing, London/New York.

Kuo H-C, Hui S, Choi J, et al. (2014) Secret lifestyles of Neurospora crassa. Sci. Rep. 4.

Langmead B, Salzberg SL (2012) Fast gapped-read alignment with Bowtie 2. Nat Meth 9, 357-359.

Le Gac M, Giraud T (2008) Existence of a pattern of reproductive character displacement inHomobasidiomycotabut not inAscomycota. Journal of Evolutionary Biology 21, 761-772.

Lesieur D, Gauthier S, Bergeron Y (2002) Fire frequency and vegetation dynamics for the south-central boreal forest of Quebec, Canada. Canadian Journal of Forest Research 32, 1996-2009.

Li H, Handsaker B, Wysoker A, et al. (2009) The sequence alignment/map format and SAMtools. Bioinformatics 25, 2078-2079.

Liti G, Carter DM, Moses AM, et al. (2009) Population genomics of domestic and wild yeasts. Nature 458, 337-341. 
Lynch M (2010) Evolution of the mutation rate. Trends in Genetics 26, 345-352.

Maynard-Smith J, Smith NH, O'Rourke M, Spratt BG (1974) The hitch-hiking effect of a favourable gene. Genetical Research 23, 23-35.

McKenna A, Hanna M, Banks E, et al. (2010) The Genome Analysis Toolkit: a MapReduce framework for analyzing next-generation DNA sequencing data. Genome Research 20, 1297-1303.

Menkis A, Bastiaans E, Jacobson DJ, Johannesson H (2009) Phylogenetic and biological species diversity within the Neurospora tetrasperma complex. Journal of Evolutionary Biology 22, 1923-1936.

Messer PW, Petrov DA (2013) Population genomics of rapid adaptation by soft selective sweeps. Trends in Ecology \& Evolution 28, 659-669.

Metzenberg R (2004) Bird medium: An alternative to Vogel medium. Fungal Genet Newsl 51, 19-20.

Nachman MW, Payseur BA (2012) Recombination rate variation and speciation: theoretical predictions and empirical results from rabbits and mice. Philosophical Transactions of the Royal Society B: Biological Sciences 367, 409-421.

Nathan G. Swenson, Daniel J. Howard (2005) Clustering of Contact Zones, Hybrid Zones, and Phylogeographic Breaks in North America. The American Naturalist 166, 581-591.

Nei M (1982) Evolution of human races at the gene level. In: Human Genetics, Part A: The Unfolding Genome (ed. B B-T), pp. 167-181. Alan R. Liss, New York.

Nei M, Li W (1979) Mathematical model for studying genetic variation in terms of restriction endonucleases. Proceedings of the National Academy of Sciences of the USA 76, 5269-5273.

Nosil P, Schluter D (2011) The genes underlying the process of speciation. Trends in Ecology \& Evolution 26, 160-167.

Nosil P, Vines TH, Funk DJ (2005) Perspective: Reproductive isolation caused by natural selection against immigrants from divergent habitats. Evolution 59, 705-719.

Novo M, Bigey F, Beyne E, et al. (2009) Eukaryote-to-eukaryote gene transfer events revealed by the genome sequence of the wine yeast Saccharomyces cerevisiae EC1118. Proceedings of the National Academy of Sciences 106, 16333-16338.

Perkins DD, Turner BC (1988) Neurospora from natural populations: toward the population biology of a haploid eukaryote. Experimental Mycology 12, 91-131.

Perkins DD, Turner BC, Barry EG (1976) Strains of Neurospora Collected from Nature. Evolution 30, 281313.

Pickrell JK, Pritchard JK (2012) Inference of population splits and mixtures from genome-wide allele frequency data. PLoS genetics 8, e1002967. 
Presgraves DC (2007) Speciation genetics: epistasis, conflict and the origin of species. Current Biology 17, R125-R127.

Racimo F, Sankararaman S, Nielsen R, Huerta-Sánchez E (2015) Evidence for archaic adaptive introgression in humans. Nature Reviews Genetics 16, 359-371.

Radic T, Gastaldello S, Diegmann J, Roenneberg T (2012) Phylogenic analysis of additional Neurospora crassa isolates. Fungal Genetics Reports 59, 13-20.

Reich D, Thangaraj K, Patterson N, Price AL, Singh L (2009) Reconstructing Indian population history. Nature 461, 489-494.

Rieseberg L, Blackman BK (2010) Speciation genes in plants. Annals of Botany 106, 439-455.

Roux C, Fraïsse C, Castric V, et al. (2014) Can we continue to neglect genomic variation in introgression rates when inferring the history of speciation? A case study in a Mytilus hybrid zone. Journal of Evolutionary Biology 27, 1662-1675.

Roux C, Tsagkogeorga G, Bierne N, Galtier N (2013) Crossing the Species Barrier: Genomic Hotspots of Introgression between Two Highly Divergent Ciona intestinalis Species. Molecular Biology and Evolution 30, 1574-1587.

Schluter D (2000) The ecology of adaptive radiation Oxford University Press.

Staben C, Yanofsky C (1990) Neurospora crassa a mating-type region. Proceedings of the National Academy of Sciences 87, 4917-4921.

Stamatakis A (2014) RAxML version 8: a tool for phylogenetic analysis and post-analysis of large phylogenies. Bioinformatics 30, 1312-1313.

Strandberg R, Nygren K, Menkis A, et al. (2010) Conflict between reproductive gene trees and species phylogeny among heterothallic and pseudohomothallic members of the filamentous ascomycete genus Neurospora. Fungal Genetics and Biology 47, 869-878.

Stukenbrock EH (2013) Evolution, selection and isolation: a genomic view of speciation in fungal plant pathogens. New Phytologist 199, 895-907.

Swenson NG, Howard DJ (2005). Clustering of contact zones, hybrid zones, and phylogeographic breaks in North America. The American Naturalist 166, 581-591.

Szpiech ZA, Jakobsson M, Rosenberg NA (2008) ADZE: a rarefaction approach for counting alleles private to combinations of populations. Bioinformatics 24, 2498-2504.

Taylor JW, Hann-Soden C, Branco S, Sylvain I, Ellison CE (2015) Clonal reproduction in fungi. Proceedings of the National Academy of Sciences 112, 8901-8908.

Taylor JW, Jacobson DJ, Kroken S, et al. (2000) Phylogenetic species recognition and species concepts in fungi. Fungal Genetics and Biology 31, 21-32. 
Tsai IJ, Bensasson D, Burt A, Koufopanou V (2008) Population genomics of the wild yeast Saccharomyces paradoxus: Quantifying the life cycle. Proceedings of the National Academy of Sciences of the United States of America 105, 4957-4962.

Turner BC, Perkins DD, Fairfield A (2001) Neurosporafrom Natural Populations: A Global Study. Fungal Genetics and Biology 32, 67-92.

Turner E, Jacobson DJ, Taylor JW (2010) Reinforced postmating reproductive isolation barriers in Neurospora, an Ascomycete microfungus. Journal of Evolutionary Biology 23, 1642-1656.

Vogel H (1956) A convenient growth medium for Neurospora (Medium N). Microb Genet Bull 13, 42-43.

Wen J (1999) Evolution of eastern Asian and eastern North American disjunct distributions in flowering plants. Annual Review of Ecology and Systematics, 421-455.

Wu Cl (2001) The genic view of the process of speciation. Journal of Evolutionary Biology 14, 851-865. 